\title{
Nuclear parton distribution functions with uncertainties in a general mass variable flavor number scheme
}

\author{
Hamzeh Khanpour ${ }^{1,2,3,{ }^{*}}$ Maryam Soleymaninia ${ }^{2, \dagger}$ S. Atashbar Tehrani® ${ }^{2, \ddagger}$ \\ Hubert Spiesberger $\odot,{ }^{4,8}$ and Vadim Guzey ${ }^{5, \|}$ \\ ${ }^{1}$ Department of Physics, University of Science and Technology of Mazandaran, \\ P.O. Box 48518-78195, Behshahr, Iran \\ ${ }^{2}$ School of Particles and Accelerators, Institute for Research in Fundamental Sciences (IPM), \\ P.O. Box 19395-5531, Tehran, Iran \\ ${ }^{3}$ Department of Theoretical Physics, Maynooth University, Maynooth, Co. Kildare, Ireland \\ ${ }^{4}$ PRISMA ${ }^{+}$Cluster of Excellence, Institut für Physik, Johannes-Gutenberg-Universität, \\ Staudinger Weg 7, D-55099 Mainz, Germany \\ ${ }^{5}$ National Research Center “Kurchatov Institute," Petersburg Nuclear Physics Institute (PNPI), \\ Gatchina 188300, Russia
}

(Received 2 October 2020; accepted 1 July 2021; published 16 August 2021)

\begin{abstract}
In this article we obtain a new set of nuclear parton distribution functions (nuclear PDFs) at next-toleading order and next-to-next-to-leading order accuracy in perturbative QCD. The common nuclear deep-inelastic scattering (DIS) data analyzed in our study are complemented by the available chargedcurrent neutrino DIS data with nuclear targets and data from Drell-Yan cross section measurements for several nuclear targets. In addition, the most recent DIS data from the Jefferson Lab CLAS and Hall C experiments are also added to our data sample. For these specific datasets, we consider the impact of target mass corrections and higher twist effects which are expected to be important in the region of large $x$ and intermediate-to-low $Q^{2}$. Our analysis is based on a publicly available open-source tool, APFEL, which has been modified to be applicable for our analysis of nuclear PDFs. Heavy-quark contributions to nuclear DIS are considered within the framework of the FONLL general-mass variable-flavor-number scheme. The most recent CT18 PDFs are used as baseline proton PDFs. The uncertainties of nuclear PDFs are determined using the standard Hessian approach. The results of our global QCD analysis are compared with existing nuclear PDF sets and with the fitted cross sections, for which our set of nuclear PDFs provides a very good description.
\end{abstract}

DOI: 10.1103/PhysRevD.104.034010

\section{INTRODUCTION}

Nuclear parton distribution functions (nuclear PDFs) [1-19] quantify the structure of quarks and gluons in nucleons bound in a nucleus and are essential ingredients for the calculation of hard scattering cross sections in charged lepton deeply inelastic scattering (DIS) off nuclear targets and high-energy heavy-ion collisions. Based on the collinear factorization theorem, the nonperturbative nuclear

\footnotetext{
*Hamzeh.Khanpour@cern.ch

†Maryam_Soleymaninia@ipm.ir

*Atashbar@ipm.ir

\$spiesber@uni-mainz.de

"guzey_va@nrcki.pnpi.ru
}

Published by the American Physical Society under the terms of the Creative Commons Attribution 4.0 International license. Further distribution of this work must maintain attribution to the author(s) and the published article's title, journal citation, and DOI. Funded by SCOAP ${ }^{3}$.
PDFs are process independent and, as in the case of free-proton PDFs, their scale dependence is governed by the standard Dokshitzer-Gribov-Lipatov-Altarelli-Parisi (DGLAP) evolution equations [20-23]. This framework has been shown to be consistent with experimental data on nuclear DIS and heavy-ion collisions at the CERN-LHC.

A precise determination of nuclear PDFs is crucial for studies of the strong interaction in high-energy scattering processes in heavy-ion collisions, such as proton-lead $(\mathrm{p}-\mathrm{Pb})$ and lead-lead $(\mathrm{Pb}-\mathrm{Pb})$ collisions at the CERNLHC. Furthermore, nuclear PDFs are important for highenergy neutrino interactions with heavy nuclear targets, which are sensitive to the separation of up- and down-type quarks, and hence, could provide important information for the decomposition of quark flavors in a QCD analysis [4].

Several collaborations have recently presented new determinations of nuclear PDFs using the available experimental data, improved theoretical assumptions, and advanced methodological settings. For the most recent 
determination of nuclear PDFs, we refer the reader to the analyses by the nNNPDF Collaboration [1,2], KA15 [3], EPPS16 [5], TUJU19 [4], RKPZ [7], AT12 [10], KP14 [11], DSSZ [12], HKNO 7 [17], and nCTEQ15 [18]. Some of the mentioned nuclear PDF determinations are based on nuclear DIS data only. By using these data alone with a rather limited kinematic coverage, significant simplifying assumptions for the nuclear PDF parametrizations need to be taken into account. Hence, the constraints on the extracted quark and gluon nuclear PDFs are rather limited in these analyses.

Nuclear PDFs at next-to-next-to-leading order (NNLO) accuracy in perturbative quantum chromodynamics (pQCD) have been studied for the first time by KA15 [3] in the zero-mass variable-flavor-number scheme. The more recent work by nNNPDF1. 0 [1] is also performed at NNLO by applying the NNPDF methodology, and the resulting nuclear PDFs are determined by a neural network in the general-mass variable-flavor-number scheme (GMVFNS). The most recent study by TUJU19 [4] was performed at NNLO accuracy as well, but was based on the open-source xFitter package [24] within the nCTEQ Collaboration framework.

It is important to perform this study considering a different framework and to define the bound nucleon PDFs relative to a free-nucleon baseline using the most recent proton PDF determination. The work presented in our paper focuses on the determination of new nuclear PDF sets, which we refer to as KSASG20, at next-to-leading order (NLO) and NNLO accuracy in pQCD. All available and up-to-date neutral-current charged lepton nuclear DIS, charged-current neutrino DIS experimental data, and the Drell-Yan (DY) cross section ratios for several nuclear targets are used. The former two datasets are sensitive to the flavor composition.

Our analysis also incorporates the most recent DIS data for several nuclei at high $x$ from the Jefferson Lab CLAS and Hall C experiments. The JLab experiments provide a wealth of nuclear DIS data in the kinematic region of large Bjorken $x$ and intermediate-to-low photon virtuality $Q^{2}$. Hence, we particularly consider the impact of target mass corrections (TMCs) and higher twist effects (HT), which are expected to be important in the kinematic range of the JLab data.

The work presented in this paper is based on the publicly available open-source APFEL package [25], which has been modified in order to accommodate the data from nuclear collisions, i.e., neutral-current charged lepton and chargedcurrent neutrino DIS on nuclear targets. For the heavyquark contributions, we use the FONLL-B and FONLL-C implementations of the GM-VFNS at NLO and NNLO, respectively. The standard "Hessian" approach is used to estimate the uncertainties of nuclear PDFs for quarks and gluons due to experimental errors. The resulting uncertainties are examined in view of the sparse kinematic coverage of the available data.
For the free-proton baseline, we use the most recent PDF analysis by CT18 [26], which is based mainly on the most recent measurements from the LHC and a variety of available world collider data. The CT18 PDFs are consistent with our assumptions and the kinematical cuts made for our nuclear PDF analysis. The nuclear PDFs presented in our study are available via the standard LHAPDF library in order to provide an open-source tool for phenomenological applications.

We should mention here that a large amount of new and precise data from the LHC in proton-lead and lead-lead collisions became recently available [27-31]. These high precision data, especially the data on $W$ and $Z$ boson production in proton-lead collisions obtained by the ATLAS and CMS Collaborations at center-of-mass energies of $5.02 \mathrm{TeV}$ and $8.16 \mathrm{TeV}$, could provide further constraints on nuclear PDFs, especially for the case of the nuclear gluon PDF. Their impact on nuclear PDFs has been extensively studied in Refs. [2,5]. In addition, an analysis of the impact of available experimental data for proton-lead collisions from Run I at the LHC on nuclear modifications of PDFs is reported in Ref. [32], where the Bayesian reweighting technique [33-35] was used. In Ref. [36] the impact of the single inclusive $D^{0}$ meson production data from LHCb [37] in proton-lead collisions on nuclear PDFs is quantified by the Hessian reweighting method.

It will be very interesting to repeat the analysis described here and determine a new set of nuclear PDFs by adding the data from proton-lead collisions at the LHC [27-31]. In terms of future work, we plan to revisit this study and consider also the hadron collider data from the LHC.

The remainder of the paper is organized as follows. In Sec. II, we briefly review the general theoretical formalism for a global QCD analysis of nuclear PDFs and our assumptions for the input parametrization. This section also describes how we include target mass corrections, higher twist effects, and heavy flavor contributions in the nuclear PDF analysis. The charged-lepton-nucleus DIS, very recent nuclear DIS data from JLab experiments, the neutrino(antineutrino)-nucleus DIS data, and the Drell-Yan cross section measurements analyzed in our study are listed and discussed in Sec. III. Then, in Sec. IV, the procedure of $\chi^{2}$ minimization and the estimation of nuclear PDF uncertainties are presented. In Sec. V, we show and discuss in detail the global fit results and compare with other nuclear PDFs available in the literature. This section also includes our discussions of the fit quality and the data theory comparison. Finally, the discussion and a summary of the main results are given in Sec. VI.

\section{THEORETICAL FORMALISM AND INPUT DISTRIBUTIONS}

This section presents the theoretical framework used in our analysis. First, we present the parametrization of the 
KSASG2 0 parton distributions of the nucleus. Then we discuss our method to include the heavy flavor contributions in the nuclear DIS processes.

\section{A. The parton distributions of the nucleus}

In this section, we present our strategy to parametrize the KSASG2 0 nuclear PDFs at the input scale. Similarly to our previous analysis [3], and as in other QCD analyses available in the literature [15,17], we will work within the conventional approach which defines the nuclear PDFs, $x f_{i}^{N / A}\left(x, Q_{0}^{2} ; A, Z\right)$, for a bound nucleon in a nucleus with the atomic mass number $A$ with respect to those for a free nucleon, $x f_{i}^{N}\left(x, Q_{0}^{2}\right)$, through a multiplicative nuclear modification factor, $\mathcal{W}_{i}(x, A, Z)$ :

$$
x f_{i}^{N / A}\left(x, Q_{0}^{2} ; A, Z\right)=\mathcal{W}_{i}(x, A, Z) \times x f_{i}^{N}\left(x, Q_{0}^{2}\right),
$$

where $i$ is an index to distinguish the distribution functions for the valence quarks $u_{v}$ and $d_{v}$, the sea quarks $\bar{d}$ and $\bar{u}$, the strange quarks $s$ and $\bar{s}$, and the gluon $g$.

Using the PDFs for a bound nucleon inside a nucleus presented in Eq. (1), one can obtain the PDFs for a general nucleus $(A, Z)$ by averaging over the number of protons and neutrons inside the nuclei. This is given by

$$
\begin{aligned}
f_{i}^{(A, Z)}\left(x, Q^{2} ; A, Z\right)= & \frac{1}{A}\left[Z f_{i}^{p / A}\left(x, Q^{2} ; A, Z\right)\right. \\
& \left.+(A-Z) f_{i}^{n / A}\left(x, Q^{2} ; A, Z\right)\right] .
\end{aligned}
$$

The bound neutron PDFs, $f_{i}^{n / A}\left(x, Q^{2} ; A, Z\right)$, are obtained from the bound proton PDFs, $f_{i}^{p / A}\left(x, Q^{2} ; A, Z\right)$, by assuming isospin symmetry in Eq. (1).

For the nuclear modification functions, $\mathcal{W}_{i}(x, A, Z)$, we follow the QCD analyses described in Refs. $[3,10,13,17$, 38-40] and assume the following cubic-type modification function:

$$
\begin{aligned}
\mathcal{W}_{i}(x, A, Z)= & 1+\left(1-\frac{1}{A^{\alpha}}\right) \\
& \times \frac{a_{i}(A)+b_{i}(A) x+c_{i}(A) x^{2}+d_{i}(A) x^{3}}{(1-x)^{\beta_{i}}} .
\end{aligned}
$$

The advantage of the cubic form with the additional term $d_{i}$ in contrast to a quadratic-type function, i.e., with $d_{i}=0$, is that the nuclear modification becomes flexible enough to accommodate both shadowing and antishadowing in the valence quark distributions. For a detailed investigation of these functions, we refer the reader to Refs. $[3,16,17]$. The explicit $A$-dependent prefactor in $\mathcal{W}_{i}$ in Eq. (3) is constructed in such a way that for the proton $(A=1)$, one recovers the underlying free-proton PDFs. The parameter $\alpha$ is considered to be fixed at $\alpha=\frac{1}{3}$. The two terms of the prefactor $1-A^{-\alpha}$ describe nuclear volume and surface contributions [16,41]. For the valence quark distributions, $\beta_{v}$ is fixed at 0.81 , and for the sea quark and gluon densities, we fix $\beta_{\bar{q}}=\beta_{g}=1$ [16].

In general, all the coefficients $a_{i}, b_{i}, c_{i}$, and $d_{i}$ could carry an $A$ - and a $Z$-dependence [3]. Since the experimental data do not provide sufficient information to perform a stable fit for such a general ansatz, we therefore consider the $d$ parameter for the gluon density to be fixed at zero, i.e., $d_{g}=0$. In order to give further and enough flexibility to our modification factor, we assume the following $A$-dependence of the parameters $a_{i}$ for sea quarks, $b_{i}$ and $c_{i}$ for all parton flavors, and $d_{i}$ for valence and sea quarks:

$$
\begin{aligned}
& a_{i}(A)=a_{i}+\left(1-\frac{1}{A^{\epsilon_{1}}}\right) \quad(i=\bar{q}), \\
& b_{i}(A)=b_{i}+\left(1-\frac{1}{A^{\epsilon_{2}}}\right) \quad(i=v, \bar{q}, g), \\
& c_{i}(A)=c_{i}+\left(1-\frac{1}{A^{\epsilon_{3}}}\right) \quad(i=v, \bar{q}, g), \\
& d_{i}(A)=d_{i}+\left(1-\frac{1}{A^{\epsilon_{4}}}\right) \quad(i=v, \bar{q}) .
\end{aligned}
$$

The nuclear and neutrino DIS and Drell-Yan experiments analyzed in this study are performed on a variety of nuclear targets, and hence, allow us to constrain such an $A$ dependence of our fit parameters. The coefficients $a_{i}(A)$ in Eq. (4) depend on the atomic number $A$, but not all of them are free parameters that can be fitted. Among them only $a_{\bar{q}}$ needs to be determined from the QCD fit. There are three constraints for $a_{u_{v}}, a_{d_{v}}$, and $a_{g}$ due to the sum rules for the nuclear charge $Z$, the baryon number $A$, and from momentum conservation $[3,17]$. The nuclear charge is given by

$$
Z=\int_{0}^{1} d x \frac{A}{3}\left[2 f_{u_{v}}^{(A, Z)}\left(x, Q_{0}^{2}\right)-f_{d_{v}}^{(A, Z)}\left(x, Q_{0}^{2}\right)\right],
$$

the baryon number is expressed as

$$
A=\int_{0}^{1} d x \frac{A}{3}\left[f_{u_{v}}^{(A, Z)}\left(x, Q_{0}^{2}\right)+f_{d_{v}}^{(A, Z)}\left(x, Q_{0}^{2}\right)\right],
$$

and finally the momentum sum rule reads

$$
\int_{0}^{1} d x \sum_{i} x f_{i}^{(A, Z)}\left(x, Q_{0}^{2}\right)=1 .
$$

We emphasize that with these prescriptions the parameters $a_{i}$ for the valence up- and down-quark distributions are different, i.e., $a_{u_{v}} \neq a_{d_{v}}$. Hence, the nuclear correction factors for the up- and down-valence distributions are not exactly the same, but are expected to be similar in shape since the other parameters in the nuclear modification 
functions, $\mathcal{W}_{u_{v}}$ and $\mathcal{W}_{d_{v}}$, are assumed to be the same. The remaining parameters in Eq. (3) are obtained by a global $\chi^{2}$ analysis.

In the KSASG2 0 nuclear PDF analysis, we use for the free-proton PDFs the most recent PDF set of CT18 [26] at the input scale $Q_{0}^{2}=1.69 \mathrm{GeV}^{2}$, i.e.,

$$
x f_{i}^{p}\left(x, Q_{0}^{2}\right)=x f_{i}^{p, \mathrm{CT} 18}\left(x, Q_{0}^{2}\right) .
$$

Considering the discussion above and following Eq. (2), the KSASG2 0 nuclear PDFs for all parton species can be explicitly written as $[3,17]$

$$
\begin{aligned}
f_{u_{v}}^{(A, Z)}\left(x, Q_{0}^{2}\right) & =\frac{1}{A} \mathcal{W}_{u_{v}}(x, A, Z)\left[Z f_{u_{v}}^{p}\left(x, Q_{0}^{2}\right)+N f_{d_{v}}^{p}\left(x, Q_{0}^{2}\right)\right], \\
f_{d_{v}}^{(A, Z)}\left(x, Q_{0}^{2}\right) & =\frac{1}{A} \mathcal{W}_{d_{v}}(x, A, Z)\left[Z f_{d_{v}}^{p}\left(x, Q_{0}^{2}\right)+N f_{u_{v}}^{p}\left(x, Q_{0}^{2}\right)\right], \\
f_{\bar{u}}^{(A, Z)}\left(x, Q_{0}^{2}\right) & =\frac{1}{A} \mathcal{W}_{\bar{q}}(x, A, Z)\left[Z f_{\bar{u}}^{p}\left(x, Q_{0}^{2}\right)+N f_{\bar{d}}^{p}\left(x, Q_{0}^{2}\right)\right], \\
f_{\bar{d}}^{(A, Z)}\left(x, Q_{0}^{2}\right) & =\frac{1}{A} \mathcal{W}_{\bar{q}}(x, A, Z)\left[Z f_{\bar{d}}^{p}\left(x, Q_{0}^{2}\right)+N f_{\bar{u}}^{p}\left(x, Q_{0}^{2}\right)\right], \\
f_{s}^{(A, Z)}\left(x, Q_{0}^{2}\right) & =f_{\bar{s}}^{(A, Z)}\left(x, Q_{0}^{2}\right)=\mathcal{W}_{\bar{q}}(x, A, Z) f_{s}^{p}\left(x, Q_{0}^{2}\right), \\
f_{g}^{(A, Z)}\left(x, Q_{0}^{2}\right) & =\mathcal{W}_{g}(x, A, Z) f_{g}^{p}\left(x, Q_{0}^{2}\right) .
\end{aligned}
$$

As one can see from our parametrizations, we have assumed flavor-dependent sea quark densities, i.e., $f_{\bar{d}}^{(A, Z)} \neq f_{\bar{u}}^{(A, Z)}$, and the small differences between them come from the underlying free-proton PDFs and the different number of protons and neutrons in different nuclei. The nuclear DIS data which we include in our analysis are not sensitive enough to constrain the sea quark flavor decomposition, but the neutrino DIS data and the Drell-Yan cross section measurements are sufficiently sensitive to the separation of up- and down-type quarks $[4,5,12,18]$. Since we use CT18 as baseline proton PDFs, the strange quark distributions in nuclei are assumed to be flavor symmetric, $f_{\bar{s}}^{(A, Z)}=f_{s}^{(A, Z)}$. We show that the parametrization presented above is sufficiently flexible to allow for a good fit quality to the available datasets.

In our analysis, we define the momentum fraction $x$ with respect to the bound nucleon, $x=Q^{2} /\left[2\left(q \cdot p_{N}\right)\right]$, where $q$ and $p_{N}$ are the photon and nucleon momenta, respectively. With this convention, $x$ is allowed to vary in the interval $0<x<A$. However, recent studies available in the literature have shown that the nuclear structure functions fall off rapidly for $x>1[42,43]$ and, hence, can be neglected $[12,18]$. Therefore, to facilitate comparisons to other analyses, we follow the same path as other nuclear QCD analyses available in the literature and neglect the $x>1$ region.

\section{B. Target mass corrections}

In this section, we describe in detail how we include target mass corrections in our analysis. TMCs are formally subleading $1 / Q^{2}$ corrections to the leading twist structure functions, where $Q^{2}$ is the squared four-momentum transfer to the hadron. TMC effects are most pronounced in the region of large $x$ and moderate-to-small values of $Q^{2}$, which coincides with the region where nuclear PDFs are not very well determined from fits to the data. Since we include the neutral-current charged lepton DIS data from Jefferson Lab Hall C [44] and CLAS [45] experiments, a reliable extraction of nuclear PDFs from these high- $x$ and low- $Q^{2} \mathrm{JLab}$ data is expected to require an accurate consideration of TMCs in our QCD analysis.

The leading contributions to the TMC have been computed in Refs. [46,47] and have been used in several studies in the literature $[6,48-53]$. The target mass corrected structure function, $F_{2}^{\mathrm{TMC}}(x, Q)$, is given by

$$
F_{2}^{\mathrm{TMC}}(x, Q)=\frac{x^{2}}{\xi^{2} \tau^{3}} F_{2}^{0}(\xi, Q)+\frac{6 \eta x^{3}}{\tau^{2}} \int_{\xi}^{1} d y F_{2}(y, Q) / y^{2},
$$

where $\eta=M_{p}^{2} / Q^{2}$ and $M_{p}$ is the mass of the proton. In the above equation, $\xi=2 x /(1+\tau)$ refers to the Nachtmann variable [54] with $\tau=1+4 \eta x^{2}$, which shows that the TMCs vanish in the limit $M_{p}^{2} / Q^{2} \rightarrow 0$. The superscript in $F_{2}^{0}(\xi, Q)$ indicates the limit, when the proton mass $M_{p}$ is set to zero, and the second term in Eq. (10) gives the convolution term. It is found that the magnitude of these corrections could be sizeable for lower values of the photon virtuality $Q \sim M_{p}$, and hence, one needs to take TMCs into account in the high- $x$ and low- $Q$ region in a QCD fit. In our analysis of the JLab data we include the leading target mass corrections due to using the Nachtmann variable and the convolution term proportional to $\eta$, but omit terms of order $O\left(\eta^{2}\right)$ which are also known [46].

\section{Higher twist corrections}

The inclusion of higher twist corrections is particularly important at high $x$ and low $Q^{2}$. To include these effects, we use the common phenomenological $x$-dependent function from the study by CJ15 [52] and other studies available in the literature [49,55-57]. It is given by

$$
F_{2}^{A}(x, Q) \rightarrow F_{2}^{(\mathrm{LT}, \mathrm{A})}(x, Q)\left[1+C_{\mathrm{HT}}(x, A) / Q^{2}\right],
$$

where $F_{2}^{(\mathrm{LT}, \mathrm{A})}$ indicates the leading twist structure function. By assuming a simple $A^{1 / 3}$ scaling of HT effects for light nuclei, the function $C_{\mathrm{HT}}(x, A)$ can be written as [58]

$$
C_{\mathrm{HT}}(x, A)=H_{0} x^{H_{1}}\left(1+H_{2} x\right) A^{1 / 3} .
$$


In fact, we will need this only for carbon. For the $H_{i}$ parameters included in $C_{\mathrm{HT}}(x, A)$, we use $\left\{H_{0}, H_{1}, H_{2}\right\}=$ $\left\{-3.28 \mathrm{GeV}^{2}, 1.92,-2.07\right\}$ from CJ15 [52] for our NLO analysis. In addition, for our NNLO analysis, we follow the study presented in Ref. [49] and use $\left\{H_{0}, H_{1}, H_{2}\right\}=$ $\left\{-1.32 \mathrm{GeV}^{2}, 1.49,-1.96\right\}$.

\section{Heavy flavor contributions}

We note that the correct treatment of heavy quark mass contributions is important for global PDF analyses. To account for the mass dependence in the KSASG2 0 nuclear PDF analysis for the charm and bottom PDFs, we treat heavy quarks within the GM-VFNS. We refer the reader to Refs. $[59,60]$ for a detailed overview. For the KSASG20 analysis we use the heavy quark schemes implemented in the public APFEL package [25]. At NLO we choose the scheme FONLL-B which implements the NLO massive scheme calculation with NLO PDFs, while at NNLO we believe that the scheme FONLL-C is the better choice since it combines the NLO massive scheme calculation with NNLO PDFs [61,62]. We refer the reader to Ref. [48] for more details of these schemes.

The CT18 proton PDFs [26], which we consider to be a baseline in our study, is based on the Aivazis-CollinsOlness-Tung (SACOT- $\chi$ ) heavy quark scheme [63,64], which is widely used in the CTEQ family of PDF fits. However, in our theoretical calculations and determination of nuclear PDFs, we use the FONLL GM-VFN scheme. In order to examine the potential mismatch between these two schemes at NLO, we have calculated the inclusive DIS cross sections for a proton target using the SACOT- $\chi$ and FONLL-B mass schemes. The results showed that the differences between these two schemes on the calculated cross sections are rather small, especially for $x>0.01$, i.e., the range which is covered by a large amount of nuclear data. In addition, the bulk of the data sets that we used in our study corresponds to cross section ratios rather than to absolute cross sections, resulting in even smaller sensitivity to the choice of the scheme. Moreover, the heavy quark production does not play a significant role in the inclusive cross sections at $x>0.2[6]$.

In summary, the choice of the heavy flavor and mass scheme is not particularly critical for our analysis and we expect that it will have a negligible effect on the calculated cross sections in the kinematic regions covered by the nuclear DIS data. We refer the reader to the findings highlighted in Ref. [6] for detailed discussions.

In order to remain consistent with the CT18 [26] baseline proton PDF analysis, for both the NLO and the NNLO fits, the heavy quark masses are fixed at $m_{c}=$ $1.30 \mathrm{GeV}$ and $m_{b}=4.75 \mathrm{GeV}$. The strong coupling constant is set equal to $\alpha_{s}\left(M_{Z}\right)=0.118$ [65] for both the NLO and NNLO fits.

\section{NUCLEAR DIS DATASETS}

In this section, we discuss in detail the datasets which we have used in the KSASG2 0 nuclear PDF analysis.

First, we start by presenting the neutral-current chargedlepton-nucleus $\left(\ell^{ \pm} A\right)$ DIS data. These include the bulk of the datasets in our analysis which help to extract wellconstrained valence and sea quark distributions; however, they provide only a limited sensitivity to the gluon distribution and to the distinction of different quark flavors. Then we discuss the charged-current neutrino(antineutrino) DIS experimental data with nuclear targets. They depend on different combinations of quark flavors, compared with the neutral-current case. The combination of neutral- and charged-current data is, however, expected to be sufficiently sensitive to the flavor composition of nonisoscalar nuclei [4]. The neutral-current charged lepton DIS data from Jefferson Lab Hall C and the most recent CLAS data measured by JLab will also be discussed. Finally, we present the Drell-Yan dilepton pair production cross section measurement by the Fermilab experiments E772 and E886, which are important to separate different quark flavors. This section also includes a detailed investigation of our data selection and kinematical cuts that need to be made when performing the KSASG2 0 global QCD analysis.

\section{A. Neutral-current charged-lepton-nucleus DIS}

The neutral-current charged $\ell^{ \pm} A$ DIS process is a powerful tool to study the nuclear structure and to extract nuclear PDFs. Hence, we consider the nuclear DIS data in the analysis as a baseline. The DIS of charged leptons off nuclear targets, which initiated all studies of nuclear PDFs, provide the best constraints on nuclear modifications of the quark densities. These data are usually presented as a ratio of structure functions for two different nuclei and span the range from 0.005 to 0.95 in momentum fraction $x$ with a maximum photon virtuality of $Q_{\max }^{2}=123 \mathrm{GeV}^{2}$. The nuclear DIS data at lower momentum fractions, namely $x<0.01$, are sensitive to the nuclear modifications of sea quarks, $\mathcal{W}_{\bar{q}}$. The data at medium-to-large $x$ mainly probe the valence quark densities. A separation between quarks and antiquarks is not possible with these data alone. Other available data such as Drell-Yan dilepton production and (anti)neutrino collisions off nuclear targets should be used to provide a discrimination between valence and sea quarks.

All available modern inclusive DIS measurements of neutral-current structure functions on nuclear targets are considered in our KSASG2 0 analysis. In particular, we use the nuclear DIS data from the NMC, EMC, and BCDMS experiments at CERN [66-73], measurements from SLAC [74-78], HERMES measurements at HERA [79], as well as the data from E665 experiment at Fermilab [80,81]. The measurements of the nuclear structure functions in such experiments are typically presented as ratios of two different nuclei, 
TABLE I. The charged lepton DIS experimental datasets for $F_{2}^{A} / F_{2}^{D}$ used in the KSASG2 0 nuclear PDF analysis. The specific nuclear targets, the experiment, the number of data points, and the related references are listed. The values of $\chi^{2}$ for the individual dataset obtained in our NLO and NNLO fits are shown as well.

\begin{tabular}{|c|c|c|c|c|c|}
\hline Nucleus & Experiment & Number of data points & $\chi_{\mathrm{NLO}}^{2}$ & $\chi_{\text {NNLO }}^{2}$ & Reference \\
\hline \multicolumn{6}{|l|}{$\mathrm{F}_{2}^{A} / \mathrm{F}_{2}^{D}$} \\
\hline \multirow[t]{2}{*}{$\mathrm{He} / \mathrm{D}$} & SLAC-E139 & 18 & 21.86 & 21.86 & [77] \\
\hline & NMC- 95 & 16 & 9.91 & 9.84 & [67] \\
\hline $\mathrm{Li} / \mathrm{D}$ & NMC- 95 & 15 & 12.16 & 12.92 & [67] \\
\hline $\mathrm{Li} / \mathrm{D}\left(\mathrm{Q}^{2}\right.$ dep. $)$ & NMC- 95 & 153 & 163.87 & 168.86 & [69] \\
\hline $\mathrm{Be} / \mathrm{D}$ & SLAC-E139 & 17 & 41.68 & 38.40 & [74] \\
\hline \multirow{5}{*}{$\mathrm{C} / \mathrm{D}$} & EMC- 88 & 9 & 8.97 & 9.13 & [68] \\
\hline & EMC- 90 & 2 & 0.13 & 0.05 & [77] \\
\hline & SLAC-E139 & 7 & 14.56 & 14.05 & [74] \\
\hline & NMC- 95 & 15 & 7.78 & 7.15 & {$[67]$} \\
\hline & FNAL -E6 65 & 4 & 3.81 & 3.50 & [81] \\
\hline C/D ( $Q^{2}$ dep. $)$ & NMC- 95 & 164 & 144.90 & 146.02 & [69] \\
\hline \multirow[t]{2}{*}{$\mathrm{N} / \mathrm{D}$} & BCDMS - 85 & 9 & 10.20 & 12.10 & [70] \\
\hline & HERMES-03 & 92 & 55.72 & 65.12 & [79] \\
\hline \multirow[t]{2}{*}{$\mathrm{Al} / \mathrm{D}$} & SLAC-E49 & 18 & 31.39 & 30.18 & {$[75]$} \\
\hline & SLAC-E139 & 17 & 7.23 & 6.64 & [74] \\
\hline \multirow[t]{4}{*}{$\mathrm{Ca} / \mathrm{D}$} & EMC- 90 & 2 & 1.96 & 1.78 & [77] \\
\hline & NMC- 95 & 15 & 30.91 & 39.48 & [67] \\
\hline & SLAC-E139 & 7 & 4.28 & 4.02 & [77] \\
\hline & FNAL-E6 65 & 4 & 5.39 & 5.99 & [81] \\
\hline \multirow[t]{4}{*}{$\mathrm{Fe} / \mathrm{D}$} & SLAC-E87 & 14 & 7.18 & 7.61 & [76] \\
\hline & SLAC-E139 & 23 & 27.58 & 25.92 & [74] \\
\hline & SLAC-E140 & 6 & 10.69 & 10.93 & [78] \\
\hline & BCDMS - 87 & 10 & 16.60 & 15.74 & [72] \\
\hline $\mathrm{Cu} / \mathrm{D}$ & EMC- 93 & 19 & 12.15 & 12.59 & [66] \\
\hline $\mathrm{Kr} / \mathrm{D}$ & HERMES-03 & 84 & 73.67 & 88.16 & [79] \\
\hline $\mathrm{Ag} / \mathrm{D}$ & SLAC-E139 & 7 & 11.12 & 14.47 & [74] \\
\hline $\mathrm{Sn} / \mathrm{D}$ & EMC -88 & 8 & 16.85 & 18.72 & {$[68]$} \\
\hline $\mathrm{Xe} / \mathrm{D}$ & FNAL-E665-92 & 4 & 3.24 & 2.84 & [80] \\
\hline $\mathrm{Au} / \mathrm{D}$ & SLAC-E139 & 18 & 31.85 & 34.77 & [74] \\
\hline $\mathrm{Pb} / \mathrm{D}$ & FNAL-E665-95 & 4 & 9.01 & 8.64 & {$[81]$} \\
\hline Total & & 781 & & & \\
\hline
\end{tabular}

$$
R\left(x, Q^{2} ; A_{1}, A_{2}\right)=\frac{F_{2}\left(x, Q^{2} ; A_{1}\right)}{F_{2}\left(x, Q^{2} ; A_{2}\right)}
$$

In Tables I and II, the measured nuclear targets used in our KSASG20 QCD analysis are listed. For each dataset, we indicate the nuclei $A_{1}$ and $A_{2}$, which are used to construct the above structure function ratios. In addition, the experiments, the corresponding number of data points after cuts, and the published references are shown as well. In order to judge the quality of the fits, the values of $\chi^{2}$ extracted from our NLO and NNLO analyses are also presented. As can be seen from Tables I and II, the number of available data points varies for different nuclei. A very large number of data points is available for the deuteron (Table I) and for heavier nuclei, such as carbon (Table II). For other nuclei, such as, e.g., lithium, only a few data points are available (Table II).
In order to remain consistent with the CT18 [26] baseline proton PDF analysis, we consider a kinematical cut on the momentum transfer $Q^{2}$ :

$$
Q^{2} \geq Q_{\min }^{2}=1.69 \mathrm{GeV}^{2}
$$

Our choice for the cut on $Q^{2}$ is the same as that of the EPS 09 [15] and EPPS16 [5] nuclear PDF analyses. We do not impose any cut on the invariant square of the final state mass, $W^{2}$, again in agreement with what was done in Refs. [5,15]. After imposing the kinematical cut on $Q^{2}$ as presented in Eq. (14), we end up with a total of $N_{\text {data }}=$ 1075 data points. As one can see from Table I, a large amount of these points correspond to ratios of heavy nuclei with respect to deuterium.

In this work, we treat the deuteron as a nucleus. Hence, in addition to the nuclear DIS data discussed above, 
TABLE II. Same as Table I, but for the charged lepton DIS datasets for $F_{2}^{A} / F_{2}^{C}$ and $F_{2}^{A} / F_{2}^{L i}$.

\begin{tabular}{|c|c|c|c|c|c|}
\hline Nucleus & Experiment & $\begin{array}{l}\text { Number of } \\
\text { data points }\end{array}$ & $\chi_{\mathrm{NLO}}^{2}$ & $\chi_{\mathrm{NNLO}}^{2}$ & Reference \\
\hline \multicolumn{6}{|l|}{$\mathrm{F}_{2}^{A} / \mathrm{F}_{2}^{C}$} \\
\hline $\mathrm{Be} / \mathrm{C}$ & NMC- 96 & 15 & 9.11 & 11.72 & [71] \\
\hline $\mathrm{Al} / \mathrm{C}$ & NMC- 96 & 15 & 5.53 & 5.54 & [71] \\
\hline \multirow{2}{*}{$\mathrm{Ca} / \mathrm{C}$} & NMC- 96 & 20 & 14.85 & 13.82 & [67] \\
\hline & NMC- 96 & 15 & 7.80 & 7.42 & [71] \\
\hline $\mathrm{Fe} / \mathrm{C}$ & NMC- 96 & 15 & 9.04 & 8.50 & [71] \\
\hline \multirow[t]{2}{*}{$\mathrm{Sn} / \mathrm{C}$} & NMC- 96 & 144 & 135.86 & 150.24 & [71] \\
\hline & NMC- 96 & 15 & 21.51 & 27.21 & [73] \\
\hline $\mathrm{Pb} / \mathrm{C}$ & NMC - 96 & 15 & 11.49 & 14.14 & [71] \\
\hline Total & & 254 & & & \\
\hline \multicolumn{6}{|l|}{$\mathrm{F}_{2}^{A} / \mathrm{F}_{2}^{\mathrm{Li}}$} \\
\hline $\mathrm{C} / \mathrm{Li}^{2}$ & NMC- 95 & 20 & 16.95 & 17.39 & [67] \\
\hline $\mathrm{Ca} / \mathrm{Li}$ & NMC- 95 & 20 & 23.49 & 25.34 & {$[67]$} \\
\hline Total & & 40 & & & \\
\hline
\end{tabular}

our analysis also includes the deuteron structure function $F_{2}^{D}$ measurements from NMC [82], BCDMS [83,84], HERMES [85], and finally the data for the deuteron-proton ratio $F_{2}^{D} / F_{2}^{p}$ from NMC [86]. The deuteron data help to extract information on the flavor asymmetric antiquark distributions, $\bar{d} \neq \bar{u}$. Therefore, these data are essential for a successful QCD fit and for extracting information on the modification factors for the deuteron. For these specific datasets, a $Q^{2} \geq 2 \mathrm{GeV}^{2}$ cut on the momentum transfer is considered. In Table III, we list the measured deuteron structure function $F_{2}^{D}$ and the deuteron-proton ratio $F_{2}^{D} / F_{2}^{p}$ used in the KSASG20 QCD analysis. After the cuts are applied, 529 data points remain, i.e., 373 for $F_{2}^{D}$ and 156 for $F_{2}^{D} / F_{2}^{p}$.

Compared with other analyses, we use a large amount of deuteron data in our fit. As one can see from Table III, the inclusion of higher-order QCD corrections significantly improves the description of NMC-96 [82] and HERMES [85] data. A slight improvement is achieved for the case of

TABLE III. Same as Table I, but for data on the deuteron structure function $F_{2}^{D}$ and the deuteron-proton ratio $F_{2}^{D} / F_{2}^{p}$.

\begin{tabular}{|c|c|c|c|c|c|}
\hline Nucleus & Experiment & $\begin{array}{l}\text { Number of } \\
\text { data points }\end{array}$ & $\chi_{\mathrm{NLO}}^{2}$ & $\chi_{\text {NNLO }}^{2}$ & Reference \\
\hline $\mathrm{D}$ & NMC- 96 & 126 & 201.90 & 88.50 & [82] \\
\hline $\mathrm{D}$ & BCDMS & 53 & 58.52 & 64.86 & [83] \\
\hline $\mathrm{D}$ & BCDMS & 155 & 245.89 & 216.84 & [84] \\
\hline $\mathrm{D}$ & HERMES & 39 & 10.28 & 5.57 & [85] \\
\hline $\mathrm{D} / \mathrm{p}$ & $\mathrm{NMC}-96$ & 156 & 148.98 & 152.71 & [86] \\
\hline Total & & 529 & & & \\
\hline
\end{tabular}

BCDMS [84] at NNLO, however, we obtained rather poor $\chi^{2}$ values both at NLO and NNLO. The $\chi^{2}$ values for the NMC - 96 [86] and the BCDMS data from Ref. [84] deserve a separate comment. Despite relatively good $\chi^{2}$ values for these specific datasets, the inclusion of higher-order QCD correction does not improve the agreement of data with theory. The variation for the $\chi^{2}$ values that can be seen in the table is possibly due to the tension among some of the datasets included in our QCD analysis, and the choice of input parametrization. It is important to note that two out of five deuteron-target data sets in Table III, namely, the BCDMS $F_{2}^{D}$ data [84] and the NMC-96 $F_{2}^{D} / F_{2}^{p}$ data [86], have already been used in the determination of the CT18 PDFs [26] that we used as a baseline. This might result in certain double fitting of nuclear PDFs. We have checked that our KSASG2 0 PDFs and the CT18 PDFs lead to very similar predictions for the deuteron structure function $F_{2}^{D}\left(x, Q^{2}\right)$ in the kinematic range of the available data which shows the internal consistency of our approach. However, a more detailed study would be required to prove that the resulting uncertainties of our KSASG2 0 nuclear PDFs are not underestimated by this double fitting issue.

The kinematic coverage of the world data for nuclear DIS used in the present global QCD analysis is shown in Fig. 1 for some selected experiments. The applied kinematic cut on nuclear DIS data is illustrated by the dashed line in the plot. The data points lying below the line are excluded in the present QCD analysis.

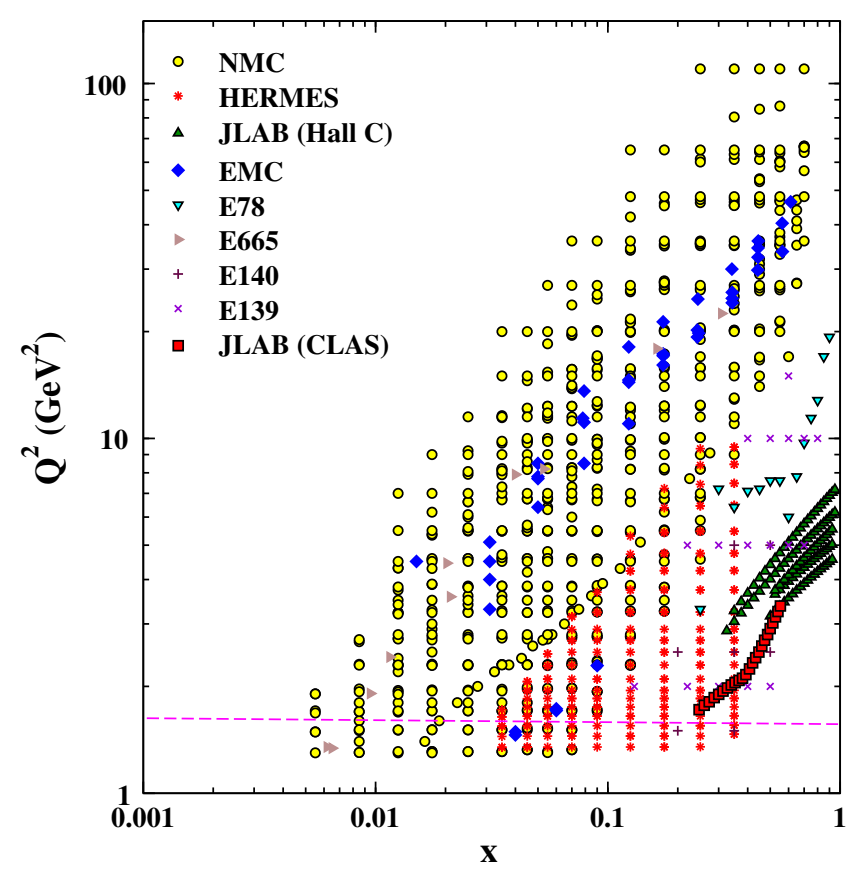

FIG. 1. The kinematic coverage of the world data for nuclear DIS data used in the present global QCD analysis. The kinematic cut, which we apply in our fit, is illustrated by the dashed line in the plot. 
TABLE IV. Neutral-current $\ell^{ \pm} A$ DIS datasets for $F_{2}^{A} / F_{2}^{D}$ from Jefferson Lab Hall C [44] and CLAS [45] at $6 \mathrm{GeV}$ [87].

\begin{tabular}{lcrrrr}
\hline \hline Nucleus & Experiment & $\begin{array}{c}\text { Number of } \\
\text { data points }\end{array}$ & $\chi_{\text {NLO }}^{2}$ & $\chi_{\text {NNLO }}^{2}$ & Reference \\
\hline $\mathrm{F}_{2}^{A} / \mathrm{F}_{2}^{D}$ & & & & & \\
$\mathrm{C} / \mathrm{D}$ & JLAB Hall C & 103 & 158.88 & 154.51 & {$[44]$} \\
$\mathrm{Pb} / \mathrm{D}$ & JLAB CLAS & 24 & 9.98 & 13.38 & {$[45]$} \\
$\mathrm{Fe} / \mathrm{D}$ & JLAB CLAS & 24 & 26.19 & 26.97 & {$[45]$} \\
$\mathrm{Al} / \mathrm{D}$ & JLAB CLAS & 24 & 16.39 & 15.23 & {$[45]$} \\
$\mathrm{C} / \mathrm{D}$ & JLAB CLAS & 24 & 14.19 & 14.66 & {$[45]$} \\
Total & & $\mathbf{1 9 9}$ & & & \\
\hline \hline
\end{tabular}

\section{B. Nuclear DIS data from JLab experiments}

JLab experiments have recently provided a wealth of nuclear DIS data in the region of large Bjorken $x$ and intermediate to low values of photon virtuality $Q^{2}$, which span a wide range of $A$. These new JLab datasets that we consider in our analysis are for $\mathrm{C}, \mathrm{Al}, \mathrm{Fe}$, and $\mathrm{Pb}$ and they could provide high-precision constraints for the nuclear PDFs in the region of low $Q^{2}$ and high $x$ (see Fig. 1). The neutral-current charged lepton DIS experimental datasets for $F_{2}^{A} / F_{2}^{D}$ from Jefferson Lab Hall C [44] and CLAS [45] measured by JLab during the $6 \mathrm{GeV}$ electron beam operation [87] are listed in Table IV. In total, the number of data points from JLab is 199 , among which 96 data points come from the CLAS Collaboration.

In our NLO and NNLO QCD analyses of the JLab DIS data, we take into account the effect of TMCs as explained above in Sec. II B, and HT corrections as presented in Sec. II C. The values of $\chi^{2}$ for the individual data for different nuclei extracted from our NLO and NNLO analyses are presented in Table IV. As one can see, the $\chi^{2}$ values presented in this table reflect the fact that the new Hall C and CLAS data measured by JLab, including the TMC and HT corrections, are described well by our QCD fits. This agrees with the results of Ref. [6], where a PDF reweighting method is used to investigate the compatibility of the available nuclear PDFs studies, EPPS16, TuJu19, and nCTEQ15, with the recently published CLAS data. Note, however, that global QCD fits, which explicitly include a particular dataset, e.g., the JLab data considered in our analysis, deliver more direct constraints on nuclear PDFs than the statistical reweighting of this data.

\section{Charged-current (anti)neutrino-nucleus DIS}

In addition to neutral-current DIS of charged leptons, we also include the data from neutrino-nucleus charged-current DIS experiments. Including the neutrino-nucleus DIS data in the analysis improves the nuclear PDF determination because it has additional sensitivity to the flavor composition of the PDFs due to the different couplings to downand up-type quarks [4].
In the KSASG2 0 nuclear PDF analysis, the cross section measurements of the CDHSW $\nu$ and $\bar{\nu} \mathrm{Fe}$ experiment [88], and the CHORUS $\nu$ and $\bar{\nu} \mathrm{Pb}$ experiment [89], have been included.

In the single- $W$ exchange approximation, the cross section for neutrino collisions with nuclear targets is given by

$$
\begin{aligned}
\frac{d^{2} \sigma^{A}}{d x d y}= & \mathcal{N}\left[\frac{y^{2}}{2} 2 x F_{1}^{m A}+\left(1-y-\frac{M_{N} x y}{2 E_{\nu}}\right) F_{2}^{m A}\right. \\
& \left. \pm y\left(1-\frac{1}{y}\right) x F_{3}^{m A}\right]
\end{aligned}
$$

where $x$ and $y$ are the standard kinematical variables for a DIS process, $m=\nu$ and $\bar{\nu}$, and $A$ denotes the nucleus. The coupling factor $\mathcal{N}$ for (anti)neutrino-nucleus collisions can be written as

$$
\mathcal{N}=\frac{G_{F}^{2} M_{N} E_{\nu}}{\pi\left(1+\frac{Q^{2}}{M_{W}^{2}}\right)^{2}} .
$$

The cross section in Eq. (15) is described in terms of three structure functions, namely $F_{1}^{m A}, F_{2}^{m A}$, and $F_{3}^{m A}$.

The structure functions for neutrino scattering in Eq. (15) are given at leading order by $[4,12,65]$

$$
\begin{aligned}
& F_{2}^{\nu A}=2 x\left(d^{A}+s^{A}+b^{A}+\bar{u}^{A}+\bar{c}^{A}\right), \\
& F_{3}^{\nu A}=2\left(d^{A}+s^{A}+b^{A}-\bar{u}^{A}-\bar{c}^{A}\right),
\end{aligned}
$$

and for antineutrino scattering by

$$
\begin{aligned}
& F_{2}^{\bar{\nu} A}=2 x\left(u^{A}+c^{A}+\bar{d}^{A}+\bar{s}^{A}+\bar{b}^{A}\right), \\
& F_{3}^{\bar{\nu} A}=2\left(u^{A}+c^{A}-\bar{d}^{A}-\bar{s}^{A}-\bar{b}^{A}\right) .
\end{aligned}
$$

Full expressions including higher-order corrections can be found in Ref. [48]. As can be seen from Eqs. (17) and (18), $F_{2}^{m A}$ is proportional to a particular nonsinglet combination of quark distributions. Hence, it is sensitive to both valence and sea quark densities. In addition, $F_{3}^{m A}$ provides additional sensitivity to the flavor composition since it depends on a different linear combination of quark and antiquark PDFs. By combining the nuclear and neutrino DIS data, one can arrive at a considerably improved valence and sea quark separation in the entire region of $x$, where the data overlap.

In addition to the CDHSW and CHORUS neutrino DIS data, there are more neutrino scattering datasets available in the literature, namely the cross sections for an iron target measured by the NuTeV Collaboration [90] and the data from the CCFR Collaboration [91]. For the CCFR measurements, the quantities $Q^{2}$ and $x$ were not publicly available for the cross sections. In addition, only the averaged structure functions $F_{2}$ and $x F_{3}$ for neutrino 
TABLE V. The $\nu A$ DIS data used in the KSASG2 0 analysis. The specific nuclear target, the experiment, the number of data points, the values of $\chi^{2}$ extracted from our NLO and NNLO fits, and the related references are listed.

\begin{tabular}{|c|c|c|c|c|c|}
\hline Nucleus & Experiment & $\begin{array}{l}\text { Number of } \\
\text { data points }\end{array}$ & $\chi_{\mathrm{NLO}}^{2}$ & $\chi_{\mathrm{NNLO}}^{2}$ & Reference \\
\hline$\nu \mathrm{Pb}$ & CHORUS & 532 & 459.71 & 569.92 & [89] \\
\hline $\bar{\nu} \mathrm{Pb}$ & CHORUS & 532 & 552.67 & 549.52 & [89] \\
\hline$\nu \mathrm{Fe}$ & CDHSW & 698 & 790.06 & 733.92 & [88] \\
\hline $\bar{\nu} \mathrm{Fe}$ & CDHSW & 696 & 695.79 & 679.00 & [88] \\
\hline Total & & 2458 & & & \\
\hline
\end{tabular}

and antineutrino scattering on iron nuclei are available, which have less sensitivity to the flavor composition [4]. Hence, we do not consider the CCFR data in our analysis.

Several studies in the literature have found some unresolved tension between the NuTeV measurements and other lepton-nucleus data [92,93]. A similar tension was also reported in Refs. [14,94] when taking into account the neutrino DIS data from the CHORUS and CCFRR measurements. Detailed studies presented in Refs. [34,95] have shown that the tension with other data was specifically due to the inclusion of data from the $\mathrm{NuTeV}$ experiment. Due to this tension, we have excluded the NuTeV neutrino DIS data from our QCD analysis.

The $\nu A$ DIS data used in our analysis are presented in Table V. The number of data points, the respective reference, and the specific nuclear target are listed as well. These datasets are subject to the same standard cut as presented in Eq. (14). In total, we include 2458 data points for neutrino-nucleus collisions from CHORUS and CDHSW. The $\chi^{2}$ values obtained in our NLO and NNLO fits are shown as well. Figure 2 shows the kinematic coverage of these data. Our kinematic cut is illustrated by the dashed line in the plot. The data points lying below the line are excluded from the present QCD analysis.

\section{Drell-Yan cross section data}

The analysis presented in our paper also incorporates Drell-Yan dilepton pair production. The expression for the differential cross section for the DY process is given in the literature [17,96-101]. The proton-nucleus differential Drell-Yan cross section can be written as a sum of two parts: the contributions of $q \bar{q}$ annihilation and the quarkgluon Compton process,

$$
\frac{d \sigma_{\mathrm{DY}}^{A}}{d Q^{2} d x_{F}}=\frac{d \sigma_{q \bar{q}}^{A}}{d Q^{2} d x_{F}}+\frac{d \sigma_{q g}^{A}}{d Q^{2} d x_{F}} .
$$

Typically, the Drell-Yan cross sections are measured in terms of the Feynman variable $x_{F}=x_{1}-x_{2}$, where the variables $x_{1}$ and $x_{2}$ refer to the momentum fractions of the involved partons. The subprocess cross sections, $d \hat{\sigma}_{q \bar{q}(0)}$

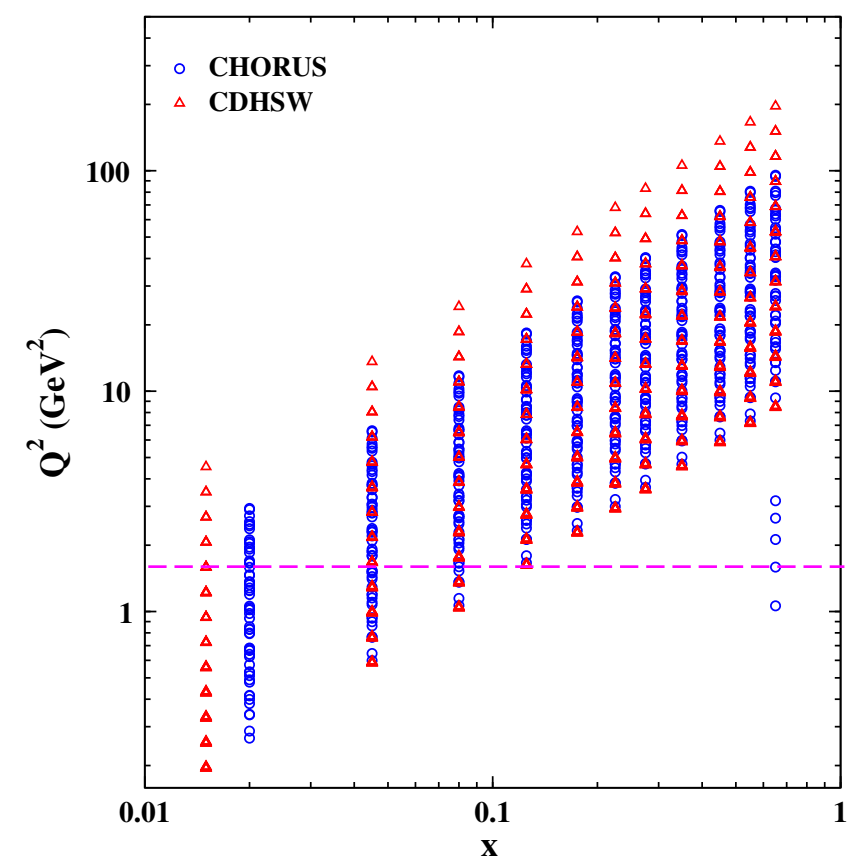

FIG. 2. The kinematic coverage of the neutrino DIS data used in the present global QCD analysis. Data points lying below the dashed line in the plot are excluded from our fit.

and $d \hat{\sigma}_{q \bar{q}(1)}$ for $q \bar{q}$ annihilation and $d \sigma_{q g}$ for $q g$ and $\bar{q} g$ scattering, combined with PDFs are given by $[17,101]$

$$
\begin{aligned}
\frac{d \sigma_{q \bar{q}}^{A}}{d Q^{2} d x_{F}}= & \frac{4 \pi \alpha^{2}}{9 Q^{2} s} \sum_{i} e_{i}^{2} \int_{x_{1}}^{1} d t_{1} \int_{x_{2}}^{1} d t_{2}\left[\frac{d \hat{\sigma}_{q \bar{q}(0)}}{d Q^{2} d x_{F}}+\frac{d \hat{\sigma}_{q \bar{q}(1)}}{d Q^{2} d x_{F}}\right] \\
& \times\left[q_{i}\left(t_{1}, Q^{2}\right) \bar{q}_{i}^{A}\left(t_{2}, Q^{2}\right)+\bar{q}_{i}\left(t_{1}, Q^{2}\right) q_{i}^{A}\left(t_{2}, Q^{2}\right)\right],
\end{aligned}
$$

$$
\begin{aligned}
\frac{d \sigma_{q g}^{A}}{d Q^{2} d x_{F}}= & \frac{4 \pi \alpha^{2}}{9 Q^{2} s} \sum_{i} e_{i}^{2} \int_{x_{1}}^{1} d t_{1} \int_{x_{2}}^{1} d t_{2} \\
& \times\left[\frac{d \hat{\sigma}_{g q}}{d Q^{2} d x_{F}} g\left(t_{1}, Q^{2}\right)\left[q_{i}^{A}\left(t_{2}, Q^{2}\right)+\bar{q}_{i}^{A}\left(t_{2}, Q^{2}\right)\right]\right. \\
& \left.+\frac{d \hat{\sigma}_{q g}}{d Q^{2} d x_{F}}\left[q_{i}\left(t_{1}, Q^{2}\right)+\bar{q}_{i}\left(t_{1}, Q^{2}\right)\right] g^{A}\left(t_{2}, Q^{2}\right)\right] .
\end{aligned}
$$

As one can see from Eqs. (20) and (21), the Drell-Yan cross sections depend on the charged-weighted sum of all quark-antiquark flavors. This is again a different combination of quark flavors compared to the total inclusive DIS cross section. Therefore the combination of DIS and DY data may, in principle, help to separately constrain up- and down-quark distributions. We should stress here that Eqs. (20) and (21) provide the DY cross section at NLO accuracy. For the calculation of the DY cross section at NNLO, we use a NNLO/NLO $K$-factor [3]. In order to obtain this $K$-factor, we have computed the NNLO DY 
TABLE VI. The Drell-Yan cross section ratios $\sigma_{\mathrm{DY}}^{A} / \sigma_{\mathrm{DY}}^{A^{\prime}}$ measured at FNAL used in the KSASG20 nuclear PDFs global fit. The specific nuclear target, the number of analyzed data points, and the related reference are listed, as well.

\begin{tabular}{lccrrr}
\hline \hline Nucleus & Experiment & Number of data points & $\chi_{\text {NLO }}^{2}$ & $\chi_{\text {NNLO }}^{2}$ & Reference \\
\hline$\sigma_{\mathrm{DY}}^{A} / \sigma_{\mathrm{DY}}^{A^{\prime}}$ & & & & & \\
$\mathrm{Fe} / \mathrm{Be}$ & FNAL-E866/NuSea & 28 & 28.38 & 28.13 & {$[104]$} \\
$\mathrm{W} / \mathrm{Be}$ & FNAL-E866/NuSea & 28 & 37.17 & 32.09 & {$[104]$} \\
$\mathrm{C} / \mathrm{D}$ & FNAL-E772-90 & 9 & 30.12 & 33.83 & {$[105]$} \\
$\mathrm{Ca} / \mathrm{D}$ & FNAL-E772-90 & 9 & 4.35 & 6.13 & {$[105]$} \\
$\mathrm{Fe} / \mathrm{D}$ & FNAL-E772-90 & 9 & 25.98 & 29.31 & {$[105]$} \\
$\mathrm{W} / \mathrm{D}$ & FNAL-E772-90 & 9 & 14.04 & 14.44 & {$[105]$} \\
Total & & $\mathbf{9 2}$ & & & \\
\hline \hline
\end{tabular}

cross sections using the DYNNLO package $[102,103]$ and then corrected the NLO cross sections by applying this $K$-factor during the fit.

The datasets for the Drell-Yan cross section ratios $\sigma_{\mathrm{DY}}^{A} / \sigma_{\mathrm{DY}}^{A^{\prime}}$ analyzed in the KSASG20 global QCD fit are listed in Table VI. The specific nuclear targets, the number of data points, the extracted $\chi^{2}$ values for the NLO and NNLO QCD analysis, and the related references are listed in this table, as well. In total, we include 92 DY data points.

\section{IV. $\chi^{2}$ MINIMIZATION AND UNCERTAINTY ESTIMATION}

The optimal values for the nuclear PDF parameters defined in Eq. (9) are extracted from the nuclear DIS and Drell-Yan data using the global function $\chi_{\text {global }}^{2}\left(\left\{\xi_{i}\right\}\right)$, given by

$$
\chi_{\text {global }}^{2}\left(\left\{\xi_{i}\right\}\right)=\sum_{m=1}^{m^{\text {exp }}} w_{m} \chi_{m}^{2}\left(\left\{\xi_{i}\right\}\right),
$$

where $m$ labels the experiment. $w_{m}$ allows us, in principle, to include datasets with different weight factors. However, we always use the default value $w_{m}=1$ for all experimental datasets [106-109]. Each experiment contributes with $\chi_{m}^{2}\left(\left\{\xi_{i}\right\}\right)$ to the global $\chi^{2}$. These terms depend on the fit parameters $\left(\left\{\xi_{i}\right\}\right)$, which are identified with the parameters of the bound proton PDFs at the initial scale. $\chi_{m}^{2}\left(\left\{\xi_{i}\right\}\right)$ is calculated as

$$
\begin{aligned}
\chi_{m}^{2}\left(\left\{\xi_{i}\right\}\right)= & \left(\frac{1-\mathcal{N}_{m}}{\Delta \mathcal{N}_{m}}\right)^{2} \\
& +\sum_{j=1}^{N_{m}^{\text {data }}}\left(\frac{\left(\mathcal{N}_{m} \mathcal{O}_{j}^{\text {data }}-\mathcal{T}_{j}^{\text {theory }}\left(\left\{\xi_{i}\right\}\right)\right.}{\mathcal{N}_{m} \Delta_{j}^{\text {data }}}\right)^{2} .
\end{aligned}
$$

Here, $j$ runs over data points, $m$ indicates a given individual dataset, and $N_{m}^{\text {data }}$ corresponds to the total number of data points in this set. In the above equation, $\mathcal{O}_{j}^{\text {data }}$ is the value of the measured data point for a given observable, and $\Delta_{j}^{\text {data }}$ is the experimental error calculated from the statistical and systematic errors added in quadrature. The theoretical predictions for each data point $j$ are represented by $\mathcal{T}_{j}^{\text {theory }}\left(\left\{\xi_{i}\right\}\right)$, which has to be calculated at the same experimental kinematic point $x$ and $Q^{2}$ using the DGLAP-evolved nuclear PDFs with given parameters $\left(\left\{\xi_{i}\right\}\right)$. We use the CERN subroutine MINUIT [110] to determine the independent fit parameters of nuclear PDFs $f_{i}^{(A, Z)}\left(x, Q^{2} ; A, Z\right)$ by minimizing $\chi_{\text {global }}^{2}\left(\left\{\xi_{i}\right\}\right)$.

In Eq. (23), $\Delta \mathcal{N}_{m}$ describes the overall normalization uncertainty for each charged lepton DIS experiment. We include the normalization $\mathcal{N}_{m}$ of different experiments as a free parameter along with other independent fit parameters $\left(\left\{\xi_{i}\right\}\right)$. First, we determine their values in a global prefit, then we fix them on their best-fitted values when we determine the uncertainties of the nuclear PDF parameters.

The quality of the QCD fit can be estimated from the resulting $\chi^{2} / N^{\text {data }}$, where $N^{\text {data }}$ indicates the number of data points.

After describing the method to obtain the central value of the KSASG20 nuclear PDFs by minimizing the $\chi_{\text {global }}^{2}\left(\left\{\xi_{i}\right\}\right)$ function, we are in a position to present our method to estimate the uncertainties of our nuclear PDFs. There are three established methods, namely the Hessian method [111,112], the Monte Carlo (MC) method [1,113], and the Lagrange multiplier method [107], which can be used for the error analysis. The analysis of the uncertainties in KSASG20 is done using the standard "Hessian" approach $[5,60,111,112]$, which we will briefly describe in the following. For the uncertainty estimate, we follow the notation adopted in Refs. [111,112] and refer the reader to these publications for a detailed discussion of the Hessian formalism.

The KSASG2 0 nuclear PDF uncertainties are estimated by using the Hessian matrix, $H$, defined by 


$$
\begin{aligned}
{\left[\delta f^{(A, Z)}\right]^{2}=} & T^{2} \sum_{m, n}\left(\frac{\partial f^{(A, Z)}(x,\{\xi\})}{\partial \xi_{m}}\right)_{\xi=\hat{\xi}} \\
& \times H_{m n}^{-1}\left(\frac{\partial f^{(A, Z)}(x,\{\xi\})}{\partial \xi_{n}}\right)_{\xi=\hat{\xi}},
\end{aligned}
$$

where $H_{m n}$ corresponds to the components of the Hessian matrix obtained by the CERN subroutine MINUIT, $\{\hat{\xi}\}$ indicates the set of optimum independent fit parameters, and $\xi_{n}$ are the fit parameters of the chosen functional form at the initial scale. The value of $T^{2}=\Delta \chi_{\text {global }}^{2}$ in Eq. (24) is the tolerance for the required confidence interval. It is calculated so that the confidence level (CL) becomes the one- $\sigma$ error range, i.e., $68 \% \mathrm{CL}$, for a given number of independent fit parameters. In an ideal case, with the standard "parameter-fitting" criterion for one free parameter, one would choose the tolerance criterion $T^{2}=$ $\Delta \chi_{\text {global }}^{2}=1$ for a $68 \%$, i.e., one- $\sigma \mathrm{CL}$, or $T^{2}=2.71$ for a 90\% CL [60]. In the KSASG2 0 nuclear PDF analysis, the tolerance for $\chi_{\text {global }}^{2}$ is based on the method presented in Refs. $[5,17,60]$. In our study with 18 free fit parameters it becomes $\Delta \chi_{\text {global }}^{2}=20$ at the $68 \% \mathrm{CL}$.

We note that other groups, e.g., nCTEQ15, EPPS16, and TUJU19, base their results on different values of the tolerance criterion: $\Delta \chi^{2}=35$ for $\mathrm{nCTEQ} 15, \Delta \chi^{2}=52$ for EPPS16, and $\Delta \chi^{2}=50$ for TUJU19.

\section{RESULTS AND DISCUSSIONS}

In the following section we present the main results and findings of our QCD analysis. We first discuss the main features of the KSASG2 0 nuclear PDF parameters. Then, we assess the stability of our NLO and NNLO results with respect to the perturbative order. We present a detailed comparison with the recent NLO and NNLO nuclear PDF analyses available in the literature. Finally, the section is concluded with a discussion of the quality of our fit results by comparing the resulting structure function ratios with the nuclear DIS experimental data; we compare our theoretical predictions with the neutrino DIS and DrellYan data as well.

\section{A. Best fit parameters}

In this work, we analyze nuclear PDFs using the CT18 proton PDF set as a baseline [26]. The nuclear modification factors in Eq. (3) are extracted from QCD fits to the nuclear and neutrino(antineutrino) DIS, and Drell-Yan data. Our best fit parameters obtained in the KSASG20 NLO and NNLO fits at the initial scale $Q_{0}^{2}=1.69 \mathrm{GeV}^{2}$ are presented in Table VII along with their errors. Values marked with an asterisk (*) in this table are fixed at the given particular value since the analyzed nuclear and neutrino DIS and Drell-Yan data could not constrain these parameters well enough. The fixed values of $\beta_{g}=1$ and $\beta_{\bar{q}}=1$
TABLE VII. Best fit parameters and uncertainties of the KSASG2 0 fits at NLO and NNLO at the initial scale

\begin{tabular}{|c|c|c|}
\hline Parameters & NLO & NNLO \\
\hline$a_{v}$ & see Table VIII & see Table IX \\
\hline$b_{v}$ & $0.350 \pm 0.05$ & $0.047 \pm 0.040$ \\
\hline$\epsilon_{b_{v}}$ & $0.014 \pm 0.005$ & $0.011 \pm 0.005$ \\
\hline$c_{v}$ & $-2.112 \pm 0.121$ & $-1.373 \pm 0.084$ \\
\hline$\epsilon_{c_{v}}$ & $-0.0005 \pm 0.008$ & $0.0001 \pm 0.008$ \\
\hline$d_{v}$ & $1.961 \pm 0.081$ & $1.520 \pm 0.057$ \\
\hline$\epsilon_{d_{v}}$ & $-0.007 \pm 0.007$ & $-0.006 \pm 0.008$ \\
\hline$\beta_{v}$ & $0.81^{*}$ & $0.81^{*}$ \\
\hline$a_{\bar{q}}$ & $-0.240 \pm 0.011$ & $-0.210 \pm 0.011$ \\
\hline$\epsilon_{a_{\bar{q}}}$ & $-0.005 \pm 0.001$ & $-0.011 \pm 0.001$ \\
\hline$b_{\bar{q}}$ & $4.731 \pm 0.254$ & $5.518 \pm 0.271$ \\
\hline$\epsilon_{b_{\bar{q}}}$ & $0.228 \pm 0.037$ & $0.176 \pm 0.030$ \\
\hline$c_{\bar{q}}$ & $-23.594 \pm 1.673$ & $-25.086 \pm 1.715$ \\
\hline$\epsilon_{c_{\bar{q}}}$ & $0.154 \pm 0.072$ & $0.121 \pm 0.064$ \\
\hline$d_{\bar{q}}$ & $29.061 \pm 2.667$ & $31.678 \pm 3.063$ \\
\hline$\epsilon_{d_{\bar{q}}}$ & $0.060 \pm 0.102$ & $0.039 \pm 0.104$ \\
\hline$\beta_{\bar{q}}$ & $1.0^{*}$ & $1.0^{*}$ \\
\hline$a_{g}$ & see Table VIII & see Table IX \\
\hline$b_{g}$ & $-2.400 \pm 0.456$ & $0.497 \pm 0.450$ \\
\hline$\epsilon_{b_{g}}$ & $0.174 \pm 0.084$ & $0.100 \pm 0.072$ \\
\hline$c_{g}$ & $6.433 \pm 0.851$ & $1.786 \pm 0.663$ \\
\hline$\epsilon_{c_{q}}$ & $0.168 \pm 0.138$ & $-0.063 \pm 0.046$ \\
\hline$d_{g}$ & $0.0^{*}$ & $0.0^{*}$ \\
\hline$\epsilon_{d_{g}}$ & $0.0^{*}$ & $0.0^{*}$ \\
\hline$\beta_{g}$ & $1.0^{*}$ & $1.0^{*}$ \\
\hline$\alpha_{s}\left(M_{Z}^{2}\right)$ & $0.118^{*}$ & $0.118^{*}$ \\
\hline$m_{c}$ & $1.30^{*}$ & $1.30^{*}$ \\
\hline$m_{b}$ & $4.75^{*}$ & $4.75^{*}$ \\
\hline$\chi^{2} /$ d.o.f & $4582.066 / 4335=1.056$ & $4548.630 / 4335=1.049$ \\
\hline
\end{tabular}
$Q_{0}^{2}=1.69 \mathrm{GeV}^{2}$. Values marked with $(*)$ are fixed in our fit.

for gluon and sea quarks, as well as the value $\beta_{v}=0.81$ for valence quark densities, is motivated by the HKN07 analysis [17]. Freeing these parameters can easily lead to unphysical fit results, and hence, we have decided to keep them fixed at this stage. As we mentioned before, the heavy quark masses are fixed at $m_{c}=1.30 \mathrm{GeV}$ and $m_{b}=4.75 \mathrm{GeV}$ to be consistent with the CT18 proton PDFs. The strong coupling constant is taken as $\alpha_{s}\left(M_{Z}\right)=0.118[65]$.

As discussed in Sec. II A, the nucleus-dependent parameters $a_{i}(A, Z)$ for the sea quark densities need to be determined from the fit to data. The parameters $a_{i}(A, Z)$ for the valence quark and gluon densities depend on the mass number $A$ and atomic number $Z$ in general, and are extracted from the three constraints in Eqs. (5), (6), and (7). The numerical values for these parameters are presented in Tables VIII and IX at NLO and NNLO accuracy, respectively. 
TABLE VIII. Values of the parameters $a_{u_{v}}, a_{d_{v}}$, and $a_{g}$ for several nuclei analyzed in this study at NLO accuracy. These parameters are obtained from the sum rules for the nuclear charge $Z$, the baryon number $A$, and momentum conservation. See Sec. II A for details.

\begin{tabular}{lllc}
\hline \hline Nucleus & \multicolumn{1}{c}{$a_{u_{v}}$} & \multicolumn{1}{c}{$a_{d_{v}}$} & $a_{g}$ \\
\hline${ }^{2} \mathrm{D}$ & 0.0111404 & 0.0111404 & 0.0870223 \\
${ }^{4} \mathrm{He}$ & 0.00952477 & 0.00952477 & 0.0560934 \\
${ }^{7} \mathrm{Li}$ & 0.00774508 & 0.00867554 & 0.0337899 \\
${ }^{9} \mathrm{Be}$ & 0.00728751 & 0.00799997 & 0.0244899 \\
${ }^{12} \mathrm{C}$ & 0.00700116 & 0.00700116 & 0.0143500 \\
${ }^{14} \mathrm{~N}$ & 0.00665067 & 0.00665067 & 0.00912677 \\
${ }^{27} \mathrm{Al}$ & 0.00505471 & 0.00527678 & -0.0115712 \\
${ }^{40} \mathrm{Ca}$ & 0.00428701 & 0.00428701 & -0.0228351 \\
${ }^{56} \mathrm{Fe}$ & 0.00332813 & 0.0037376 & -0.0318663 \\
${ }^{63} \mathrm{Cu}$ & 0.00304467 & 0.00349631 & -0.0349004 \\
${ }^{84} \mathrm{Kr}$ & 0.00222106 & 0.0030206 & -0.0420497 \\
${ }^{108} \mathrm{Ag}$ & 0.00171484 & 0.00242832 & -0.0479941 \\
${ }^{119} \mathrm{Sn}$ & 0.00141338 & 0.00228757 & -0.0502205 \\
${ }^{131} \mathrm{Xe}$ & 0.00115708 & 0.00211297 & -0.0523868 \\
${ }^{197} \mathrm{Au}$ & 0.000212695 & 0.00126285 & -0.0611757 \\
${ }^{208} \mathrm{~Pb}$ & 0.000055209 & 0.00117403 & -0.0622995 \\
\hline \hline
\end{tabular}

TABLE IX. Same as Table VIII, but at NNLO accuracy.

\begin{tabular}{lccc}
\hline \hline Nucleus & $a_{u_{v}}$ & $a_{d_{v}}$ & $a_{g}$ \\
\hline${ }^{2} \mathrm{D}$ & 0.0332173 & 0.0332173 & -0.245614 \\
${ }^{4} \mathrm{He}$ & 0.0319167 & 0.0319167 & -0.258804 \\
${ }^{7} \mathrm{Li}$ & 0.0303139 & 0.0313825 & -0.268223 \\
${ }^{9} \mathrm{Be}$ & 0.0299825 & 0.0308045 & -0.272104 \\
${ }^{12} \mathrm{C}$ & 0.0298786 & 0.0298786 & -0.276295 \\
${ }^{14} \mathrm{~N}$ & 0.0295949 & 0.0295949 & -0.278435 \\
${ }^{27} \mathrm{Al}$ & 0.0282598 & 0.0285215 & -0.286744 \\
${ }^{40} \mathrm{Ca}$ & 0.0276775 & 0.0276775 & -0.291109 \\
${ }^{56} \mathrm{Fe}$ & 0.0268174 & 0.0273072 & -0.294503 \\
${ }^{63} \mathrm{Cu}$ & 0.0265775 & 0.0271192 & -0.295617 \\
${ }^{84} \mathrm{Kr}$ & 0.0258313 & 0.0267962 & -0.298186 \\
${ }^{108} \mathrm{Ag}$ & 0.0254344 & 0.0263003 & -0.300247 \\
${ }^{119} \mathrm{Sn}$ & 0.025152 & 0.0262153 & -0.301002 \\
${ }^{131} \mathrm{Xe}$ & 0.0249232 & 0.0260883 & -0.301725 \\
${ }^{197} \mathrm{Au}$ & 0.0241236 & 0.0254157 & -0.304532 \\
${ }^{208} \mathrm{~Pb}$ & 0.0239777 & 0.025356 & -0.304877 \\
\hline \hline
\end{tabular}

Regarding the best fit parameters and their errors listed in Table VII, some comments are in order. The obtained parameters for the nuclear valence quark distributions reflect the fact that the nuclear DIS data analyzed in this study constrain these distributions well enough. In addition, neutrino DIS and Drell-Yan data also play an important role in obtaining a consistent behavior for the up- and downvalence quarks. As can be seen from Table VII, some fit parameters of our sea quark and gluon densities come with larger errors, especially $\epsilon_{d_{\bar{q}}}$ and $\epsilon_{c_{g}}$. In addition, we fixed $d_{g}$ to zero. The nuclear DIS and Drell-Yan data only loosely constrain the gluon nuclear modifications because they cover a too limited range in $Q^{2}$.

To further constrain the nuclear sea quark and gluon densities and reduce their uncertainties, other observables will have to be taken into account. Once more data are included, for example the data from the LHC and a future $e A$ collider such as LHeC or FCC-he, it should be possible to relax some of the assumptions mentioned above.

\section{B. KSASG2 0 nuclear PDFs and their uncertainties}

In the following, we discuss the KSASG2 0 nuclear PDFs including the nuclear modification functions and present a detailed comparison between our NLO and NNLO analyses.

In Fig. 3, we show representations of different types of nuclear modification functions for some selected nuclei, deuterium (D), beryllium (Be), iron ( $\mathrm{Fe})$, and gold $(\mathrm{Au})$, at the scale $Q^{2}=2 \mathrm{GeV}^{2}$. The nuclear modification functions are shown for the valence quarks $\mathcal{W}_{u_{v}}$ and $\mathcal{W}_{d_{v}}$, sea quark $\mathcal{W}_{\bar{q}}$, and gluon $\mathcal{W}_{g}$ at NLO (top row) and NNLO (bottom row). We repeat here that, in this work, we have treated deuterium as a nucleus in the fitting procedure. Hence, as one can see from Fig. 3, small deviations from the baseline CT18 proton PDFs are found for deuterium. The deviations from the CT18 PDFs become larger with increasing atomic mass, and significant effects are found for heavier nuclei, such as gold. We should notice here that our results in the small- $x$ region, i.e., $x<10^{-2}$, are not directly constrained by the nuclear and neutrino DIS and Drell-Yan data, but determined by extrapolation based on our parametrization.

As can be seen from Fig. 3, the typical nuclear modification effects, such as antishadowing, shadowing, and EMC suppression, are visible at the $Q^{2}=2 \mathrm{GeV}^{2}$ for the up- and down-valence quark modifications, $\mathcal{W}_{u_{v}}$ and $\mathcal{W}_{d_{v}}$. For the NLO analysis, the nuclear modification for the gluon shows a rapid rise with increasing $x, x>0.1$. This trend repeats itself for the NNLO analysis, a behavior which is similar to what one can observe in the analyses by HKMO1 [16], HKNO 7 [17], and KA15 [3]. This behavior may be an artifact due to the used parametrization in these analyses. Figure 3 also shows that for the case of sea quarks one can observe the typical nuclear modifications. However, the magnitude of these effects slightly differs at different perturbative orders. Our nuclear modifications for quark and gluon densities are flat in the small- $x$ region. This behavior is similar to the analysis of HKN07 [17].

We continue with the discussion of the nuclear modification factors and their uncertainties for the case of lead $(\mathrm{Pb})$ as an example of a large nucleus. Lead is particularly relevant for the present and future heavy-ion program at the LHC for $\mathrm{p}-\mathrm{Pb}$ and $\mathrm{Pb}-\mathrm{Pb}$ collisions. The nuclear modification factors for lead along with their uncertainties at 

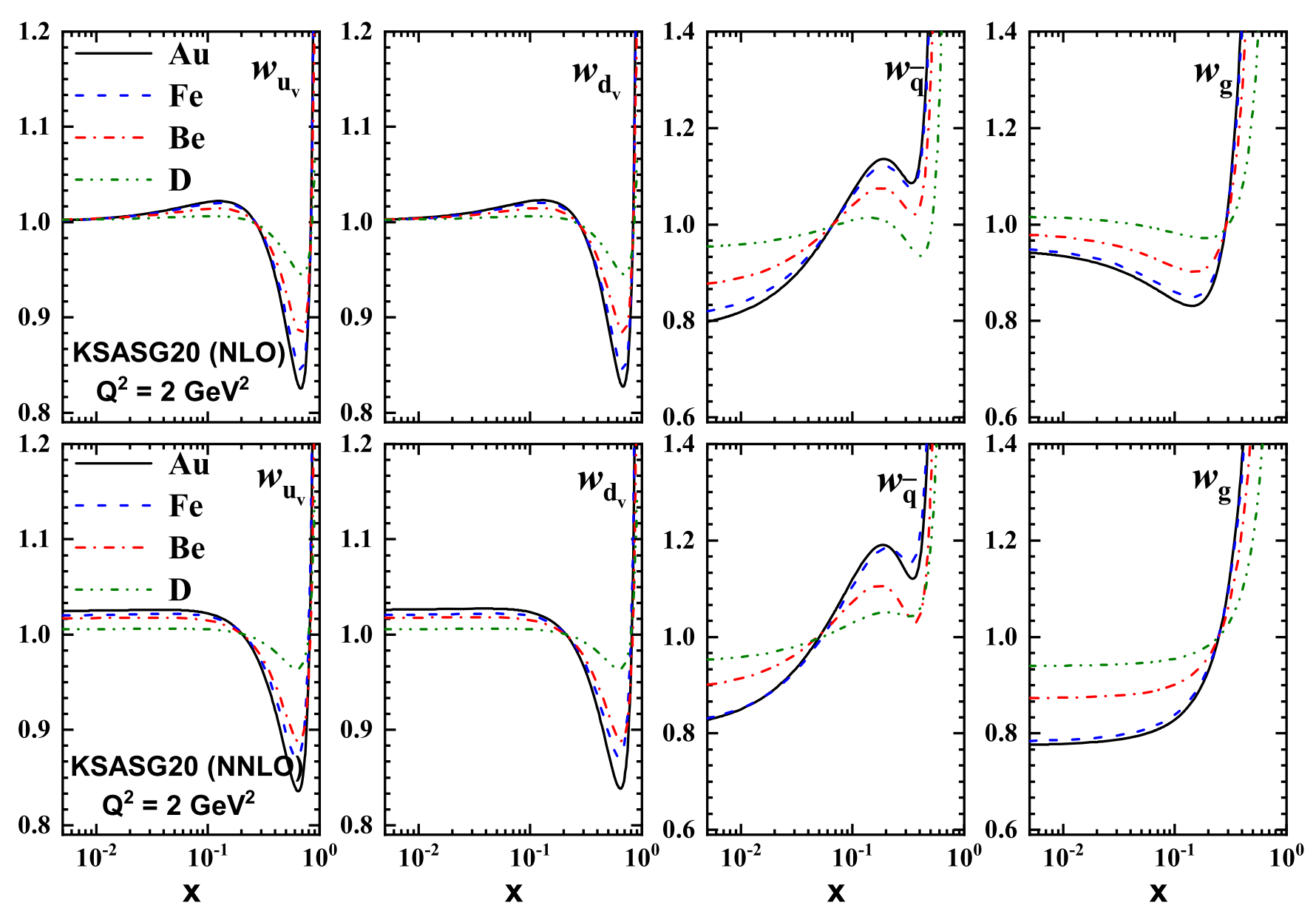

FIG. 3. The nuclear modification factors for deuterium (D), Beryllium (Be), iron (Fe), and gold (Au) at NLO (top row) and NNLO (bottom row) accuracy in pQCD and at the scale $Q^{2}=2 \mathrm{GeV}^{2}$.

$68 \%$ CL with $\Delta \chi^{2}=20$ are shown in Fig. 4. The results are shown at the scale $Q^{2}=2 \mathrm{GeV}^{2}$ at NLO and NNLO accuracy. The uncertainty bands at very small and large $x$ are not directly constrained by data. They are affected by the restricted flexibility of the considered parametrization and the limitations of the fitting framework. Due to the limited sensitivity of the available nuclear data to the sea quark and gluon densities, one has to limit the number of shape parameters. One can expect that additional new data will provide better constraints and one can envisage to consider more fit parameters allowing a larger flexibility of the modification functions.

In the following we discuss the effect of TMC and HT corrections on the fit quality and the shape of the extracted nuclear PDFs. At NLO, we observe that the inclusion of TMCs slightly increases $\chi^{2} / N_{\text {dof }}$ from 1.02 to 1.06. This can be traced back to the JLab Hall C and CLAS data. Including the HT corrections of Sec. II C yields a small improvement: $\chi^{2} / N_{\text {dof }}$ is reduced from 1.06 to 1.05 and the TMC and TMC + HT fits are quite similar. The same behavior is seen in the case of the NNLO fit. We conclude that the inclusion of TM and HT corrections is not crucial for a good description of the presently available high- $x$ data [6].

The effect of the inclusion of TMC and HT on the extracted nuclear PDFs is apparent from Fig. 5, where we compare our fit without TMC and HT (KSASG20 without TMC+HT) with our final fit, which includes both of these corrections (KSASG20). The first observation emerging from the comparisons presented in this plot is that while the TMC and HT affect the large $x$ region, they do not significantly change the results at small $x$. As one can see, the KSASG2 0 valence quark PDF is slightly larger than KSASG2 0 without TMC+HT, and this effect is mostly localized at large values of $x$, as expected. Also the gluon and the charm quark PDFs are modified by TMC + HT effects at large, while somewhat suppressed at intermediate values of $x$.

The resulting nuclear PDFs are presented in Fig. 6 for iron (left) and lead (right) at $Q^{2}=10 \mathrm{GeV}^{2}$ to show the effects of the $Q^{2}$ evolution. As we mentioned in Sec. II A, the up- and down-nuclear PDFs have been assumed to be flavor dependent, i.e., $x \bar{d} \neq x \bar{u}$. For the strange quark distributions in the nuclei, we assume, as usual, $x s=x \bar{s}$. 

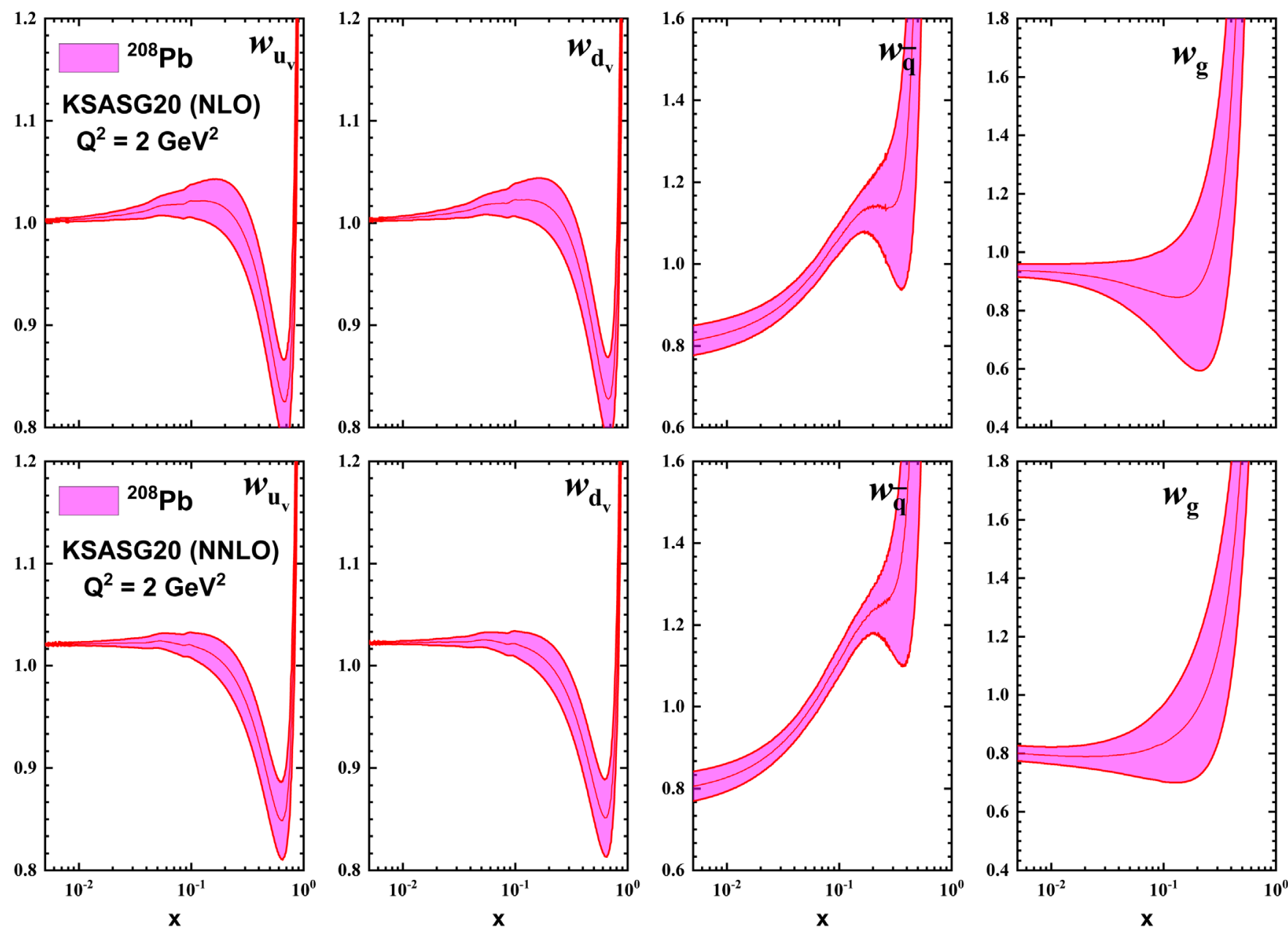

FIG. 4. The nuclear modification factors for lead at NLO and NNLO accuracy and at $Q^{2}=2 \mathrm{GeV}^{2}$. The bands show the $68 \%$ uncertainty estimation with $\Delta \chi^{2}=20$ obtained using the Hessian method.

The perturbatively generated heavy quark densities, $x \bar{c}$ and $x \bar{b}$, are obtained through DGLAP evolution. As can be seen in the figure, all the gluon and sea quark densities come with relatively large error bands at small $x$, reflecting the fact that there are not enough data constraints below $x \sim 0.01$. We find only very small differences for the fitted $\bar{u}$ and $\bar{d}$ nuclear PDFs; the corresponding error bands shown in Fig. 6 are difficult to distinguish. The small differences between $\bar{u}$ and $\bar{d}$ nuclear PDFs mainly come from the underlying free-proton PDFs and the different number of protons and neutrons in different nuclei. From our definition, the $\bar{u}$ and $\bar{d}$ PDFs are equal for isoscalar nuclei such as calcium and carbon. To show the flavor asymmetry, in Fig. 7 we present the ratio $\left(\bar{u}^{A}-\bar{d}^{A}\right) /\left(u^{A}+d^{A}\right)$ for lead $(\mathrm{Pb})$, iron $(\mathrm{Fe})$, aluminum (Al), and lithium $(\mathrm{Li})$ at $Q^{2}=10 \mathrm{GeV}^{2}$.

In the following, we compare our NLO and NNLO results. In Fig. 8, the NLO and NNLO nuclear PDFs are compared at $Q^{2}=10 \mathrm{GeV}^{2}$ for the valence quark $x q_{v}$, gluon $x g$, strange quark $x \bar{s}$, sea quarks $x \bar{u}$ and $x \bar{d}$, and finally the perturbatively generated charm quark $x \bar{c}$ density.
It is worth noticing here that the magnitude and the shape of the nuclear PDFs for a given flavor at some arbitrary scale $Q^{2}$ depends on the chosen set of reference PDFs for the free proton. Due to the limitations of the applied fitting framework and the limited sensitivity of the analyzed nuclear and neutrino(antineutrino) DIS, and the DrellYan data to the gluon and sea quark nuclear PDFs, the provided uncertainty bands are rather large, especially for small values of $x$.

A few remarks concerning the comparison between our NLO and NNLO analyses are in order. For both lead and gold nuclei, the valence quarks, $x u_{v}$ and $x d_{v}$, and strange quark, $x \bar{s}$, PDF densities at NLO and NNLO accuracy are very similar in size. The sea quark densities, $x \bar{u}$ and $x \bar{d}$, are slightly different at NLO and NNLO accuracy in the region of small $x, x<0.01$. A significant difference can be found for the gluon $x g$ and the perturbatively generated charm quark $x \bar{c}$ density at NLO and NNLO accuracy. As can be seen from Fig. 8, the NNLO gluon and charm quark PDFs are smaller than at NLO at small values of $x$. 

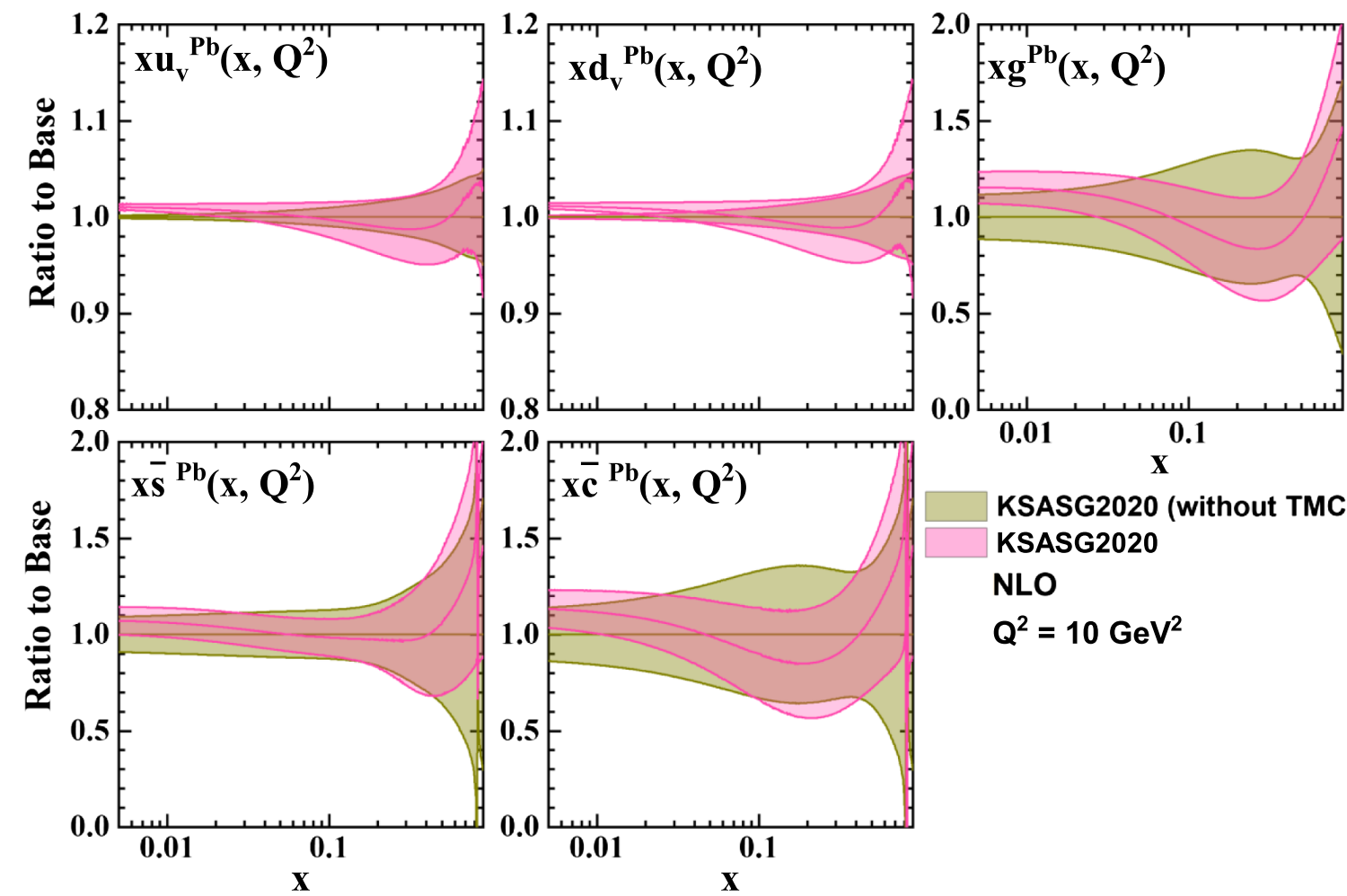

KSASG2020 (without TMC+ HT)

KSASG2020

NLO

$Q^{2}=10 \mathrm{GeV}^{2}$

FIG. 5. The ratio of KSASG20 nuclear PDFs at NLO which include TMC and HT effects compared to the corresponding results without such corrections for lead at $Q^{2}=10 \mathrm{GeV}^{2}$. We also show the uncertainty bands computed with the Hessian method.

To quantify the magnitude of NNLO corrections, we also present ratios of nuclear PDFs obtained in the NNLO fit over those of the NLO fit. This comparison is shown in Fig. 9 for lead. The results are displayed at the scale $Q^{2}=10 \mathrm{GeV}^{2}$ and we include the one- $\sigma$ uncertainty bands for $\Delta \chi^{2}=20$. As can be seen, the uncertainty for the nuclear gluon density slightly decreases when going from NLO to NNLO accuracy due to the improved overall fit quality when higher-order QCD calculations are taken into account. However, the differences between NLO and

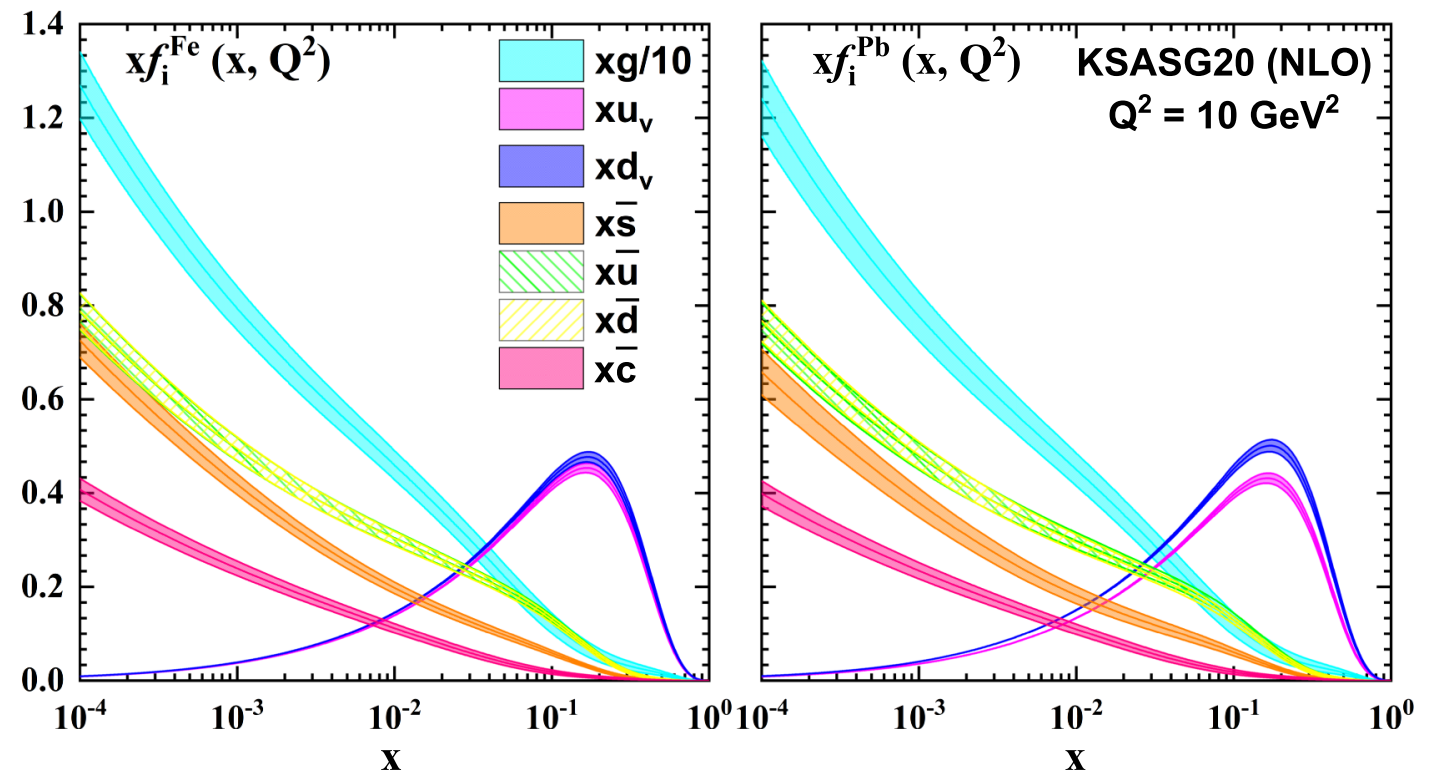

FIG. 6. KSASG2 0 nuclear PDFs and their uncertainties at $Q^{2}=10 \mathrm{GeV}^{2}$ for iron (left) and lead (right). The error bands correspond to the uncertainty estimates at $68 \% \mathrm{CL}$ with $\Delta \chi^{2}=20$ obtained using the Hessian method. 


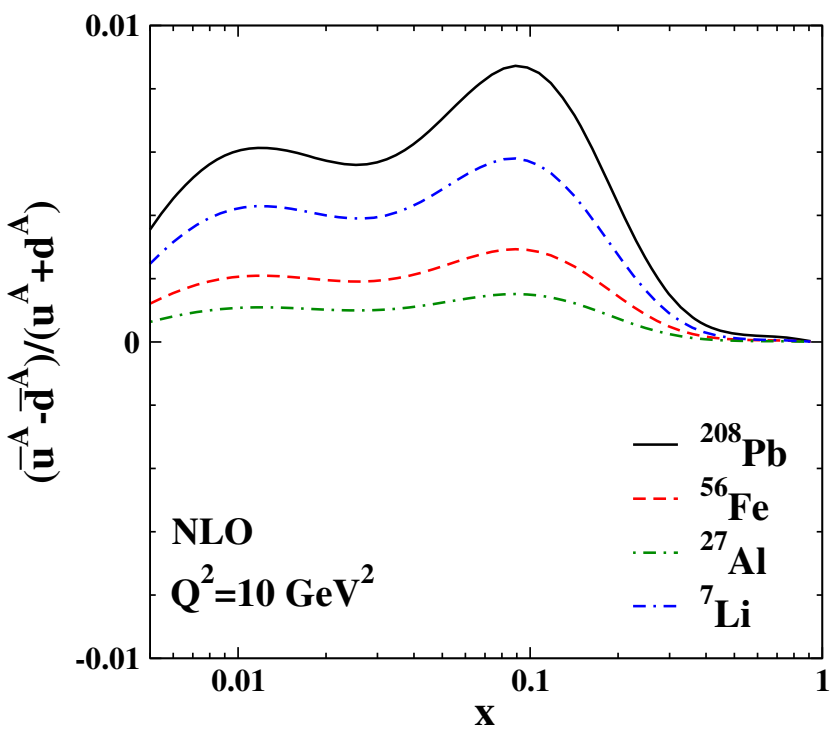

FIG. 7. The flavor asymmetry of sea quark distributions, $\left(\bar{u}^{A}-\bar{d}^{A}\right) /\left(u^{A}+d^{A}\right)$, for lead, iron, aluminum, and lithium at $\mathrm{Q}^{2}=10 \mathrm{GeV}^{2}$.

NNLO nuclear PDFs are rather small for all other parton species. These findings are consistent with the perturbative convergence of the global $\chi^{2}$ discussed in Sec. IV and listed in Tables I, II, III, V, IV, and VI. Concerning the fit quality of the total nuclear and neutrino DIS, and the Drell-Yan datasets, the most noticeable feature is a small improvement upon the inclusion of higher-order QCD corrections. The inclusion of NNLO QCD corrections affects the nuclear PDFs uncertainties and improves the description of the data as well.

\section{Comparison with other nuclear PDF sets}

In this section, we present a comparison with the most recent nuclear PDF determinations available in the literature, namely nCTEQ15 [18], EPPS16 [5], and TUJU19 [4]. Since the nCTEQ15 and EPPS16 analyses were performed only at NLO accuracy, we limit the comparison to this perturbative order. All the comparisons presented in this section have been generated by using the standard LHAPDF6 library [114] and the published grids.

Each of these nuclear PDF analyses is based on a set of assumptions; for example, the form of the input parametrization at the initial scale, the choice of the proton baseline PDFs, the included datasets and the kinematical cuts applied to the data, the perturbative order, and the scheme for the heavy quark contributions.

In order to compare our results with other nuclear PDF sets, we begin with the detailed comparisons of nuclear
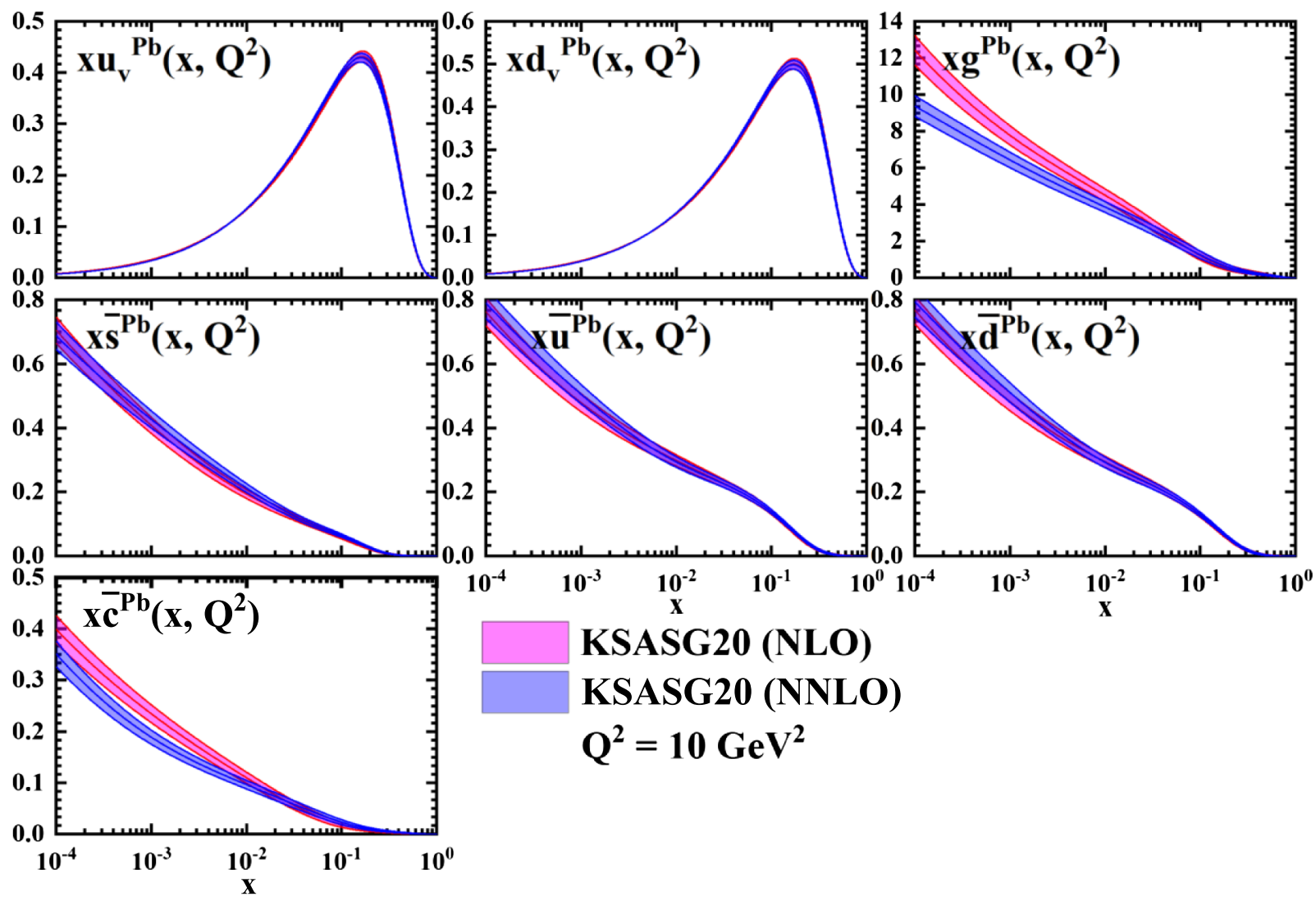

KSASG20 (NLO)

KSASG20 (NNLO)

$Q^{2}=10 \mathrm{GeV}^{2}$

FIG. 8. The nuclear PDFs at $Q^{2}=10 \mathrm{GeV}^{2}$ for lead at NLO and NNLO accuracy. The uncertainty bands have been calculated with $\Delta \chi^{2}=20$ as described in the text. 


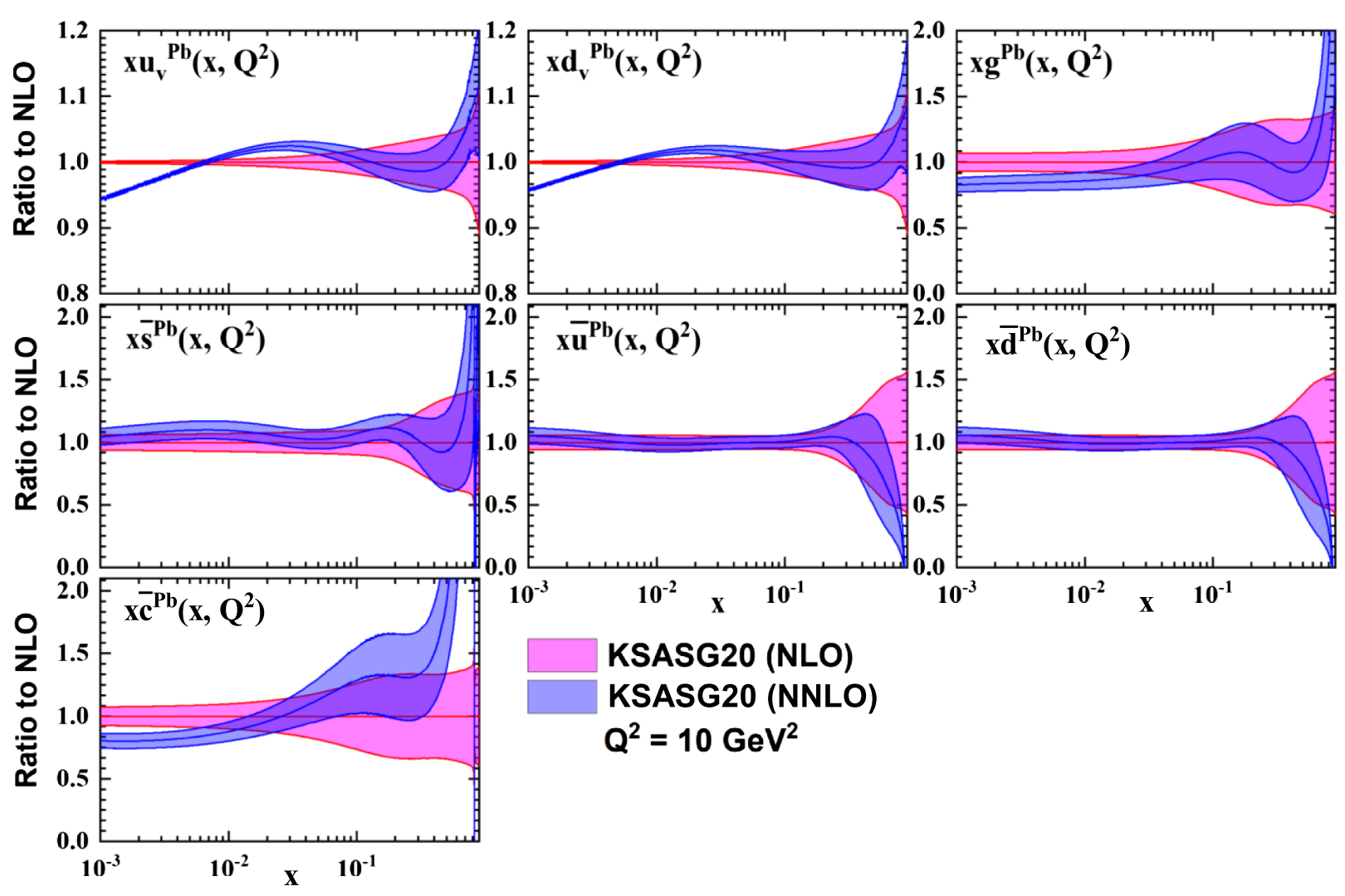

FIG. 9. Comparison between the NLO and NNLO nuclear PDFs together with their one- $\sigma$ uncertainties. The results are shown as ratios $\mathrm{NNLO} / \mathrm{NLO}$ at the scale $Q^{2}=10 \mathrm{GeV}^{2}$ for lead.

modifications in lead. In Fig. 10, we compare the KSASG2 0 nuclear modification factors in lead at NLO accuracy to those of nCTEQ15 [18], TUJU19 [4], and EPPS16 [5] at $Q^{2}=100 \mathrm{GeV}^{2}$. The comparison is presented per parton flavor $i$. The bands for KSASG2 0 show the $68 \%$ uncertainty estimation with $\Delta \chi^{2}=20$ obtained using the Hessian method. One should keep in mind that the nCTEQ15 [18] analysis is based on the tolerance criterion $\Delta \chi^{2}=35$, while in the analysis by TUJU19 [4] the condition $\Delta \chi^{2}=50$ is used and EPPS16 [5] have performed their error calculation for $\Delta \chi^{2}=52$. As one can see in Fig. 10, in the case of valence up-quarks we find a behavior similar to $\mathrm{nCTEQ} 15$ over the small and intermediate values of $x$, but a stronger large $x$ suppression.

In the case of down-valence and gluon modifications, differences both in shape and uncertainty bands can be seen between KSASG2 0 and $\mathrm{nCTEQ} 15$. The obtained valence modifications for KSASG2 0 are very similar both in shape and error bands. For the case of strange quark modification, the result is compatible with nCTEQ15 within the estimated uncertainties at medium values of $x$. A comparison of KSASG20 with TUJU19 is also presented in Fig. 10. The up-valence and sea quark modifications for the two fits can be considered compatible since the error bands always overlap over the whole range of $x$. For the sea quark, the TUJU19 uncertainties appear clearly larger than those of
KSASG20. As can be seen, the central values for the gluon and down-valence are rather different. For both distributions, the TUJU19 uncertainties are clearly larger than those of KSASG20, except in the small- $x$ region for the gluon modification. Our smaller uncertainties may be due to the fact that our parametrization is less flexible, especially in the case of the gluon density. In comparison with EPPS16, we find several differences and similarities. As one can see from Fig. 10, the uncertainty for the sea quark density for EPPS16 is much larger than the one of KSASG2 0, and also larger than the results of other groups. For the gluon density we find compatible results at intermediate and small values of $x$, but KSASG2 0 and EPPS16 are different both in shape and central value at high $x$. For the case of valence quark nuclear modifications, one can again see that the central values are compatible only for $x<0.1$. The valence quark uncertainty bands for KSASG2 0 are tighter than for EPPS16.

In spite of some differences for the nuclear modification factors, when calculating the total nuclear PDFs (see the next section), we find good agreement with the other analyses available in the literature.

For the full nuclear PDFs, we begin with a detailed comparison with the most recent nuclear PDF determination by TUJU19. Regarding the experimental DIS data to determine the nuclear PDFs, both KSASG2 0 and TUJU19 

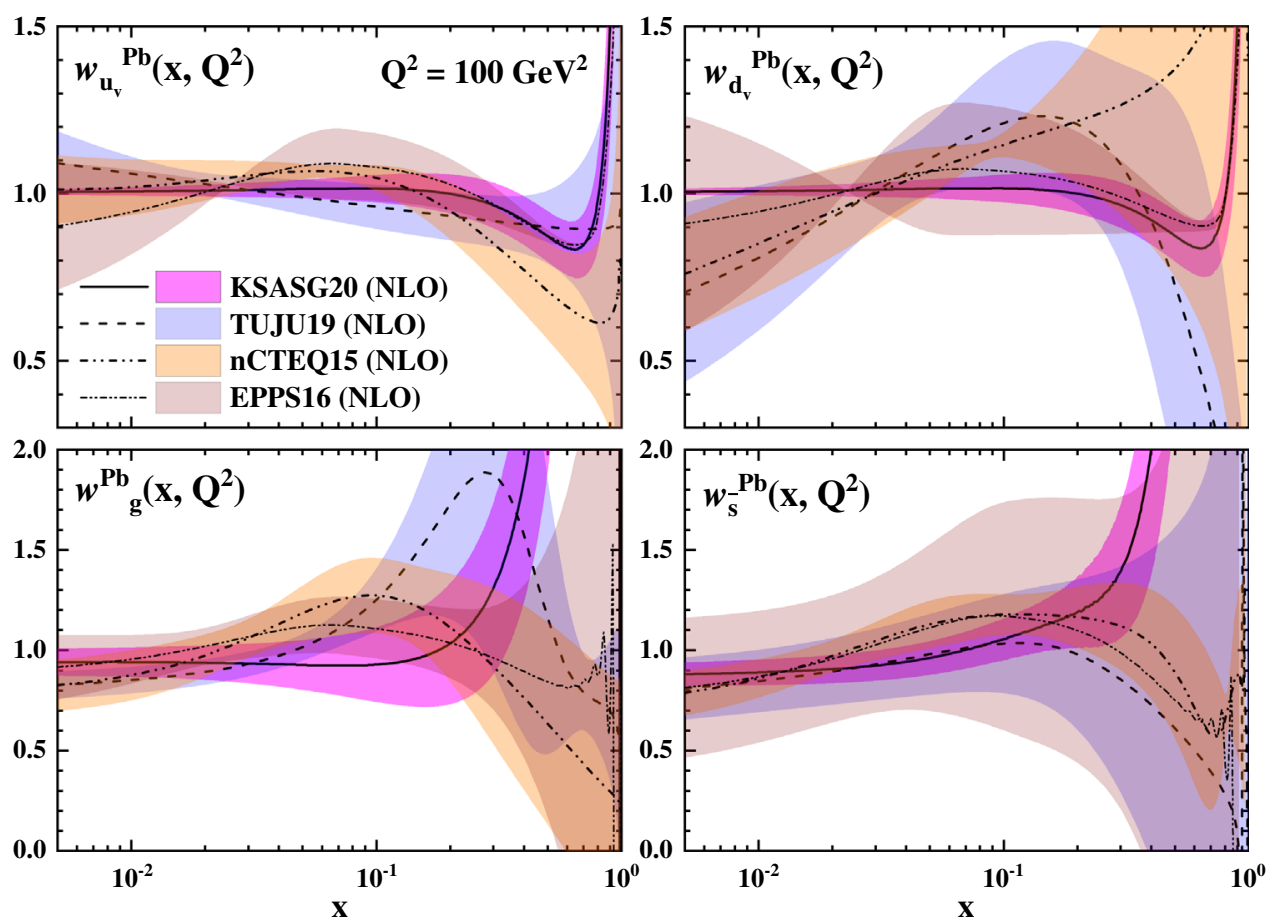

FIG. 10. The nuclear modification factors KSASG20 in lead at NLO accuracy compared to the nuclear PDF sets nCTEQ15 [18], TUJU19 [4], and EPPS16 [5] shown at the scale $Q^{2}=100 \mathrm{GeV}^{2}$. The comparison is presented per parton flavor $i$. The bands for the KSASG2 0 show the $68 \%$ uncertainty estimation with $\Delta \chi^{2}=20$ obtained using the Hessian method.

are based on the same datasets with different kinematical cuts. However, in addition to the deuteron structure function $F_{2}^{D}$ from NMC [82], we also enrich our analysis with the $F_{2}^{D}$ data from BCDMS $[83,84]$ and HERMES [85], and data for the ratio $F_{2}^{D} / F_{2}^{p}$ from NMC [86]. Our KSASG2 0 analysis also incorporates the data from Drell-Yan cross section ratios for several nuclear targets $[104,105]$ and the most recent DIS data from the Jefferson Lab CLAS and Hall C experiments $[44,45]$. For the JLab data, target mass corrections and higher twist effects are taken into account in KSASG20. The TUJU19 nuclear PDF sets are based on a CTEQ proton baseline fitted within the same framework. TUJU1 9 also assumed flavor symmetric sea quark densities, i.e., $\bar{u}=\bar{d}=s=\bar{s}$. The uncertainties for both analyses are obtained using the Hessian method, and TUJU19 calculated the uncertainty for $\Delta \chi^{2}=50$.

The comparison with TUJU19 is presented in Fig. 11 at $Q^{2}=100 \mathrm{GeV}^{2}$ for lead at NNLO accuracy. Concerning the shapes of these nuclear PDFs, a number of interesting differences between the two sets can be seen from the comparison presented in this figure. Small disagreements are found for the valence and sea quark densities, however, the two sets still agree at the one- $\sigma$ level. A moderate difference is observed for the strange quark density below $x<0.1$. A more pronounced difference in shape is observed for the gluon, charm, and bottom quark PDFs, for which the KSASG2 0 distributions are more suppressed at medium-to-small values of $x$. The differences in shape among these three densities are more marked in the case of the gluon density and bottom quark PDFs. One should remember that neither of these analyses includes data which are directly sensitive to the gluon distribution. The origin of the differences between KSASG2 0 and TUJU19, at medium-to-low $x$ for the gluon and sea quark densities, and for medium $x$ in the case of valence quark PDFs, is likely to be due to the input parametrization and a larger number of data points for the deuteron included in the KSASG2 0 analysis. Another origin of these differences is due to the inclusion of Drell-Yan data and the most recent nuclear DIS data from JLab in our analysis.

In Figs. 12 and 13, our nuclear PDFs at the scale $Q^{2}=100 \mathrm{GeV}^{2}$ are presented for lead at NLO accuracy. The most recent NLO nuclear PDF determinations available in the literature, namely from nCTEQ15 [18], EPPS16 [5], and TUJU19 [4] are also shown for comparison. We should mention here that the nCTEQ15 analysis is based on the tolerance criterion $\Delta \chi^{2}=35$, while EPPS16 presented their results for $\Delta \chi^{2}=52$. The uncertainty bands for the KSASG20 nuclear PDFs are obtained using the Hessian method with $\Delta \chi^{2}=20$, and are related to the parameters in nuclear modification factors.

In the EPPS16 analysis, the bound nucleon PDFs are defined relative to the free nucleon baseline CT14 PDFs [115], as for the case of our previous study KA15 [3] where we considered the same framework and used 

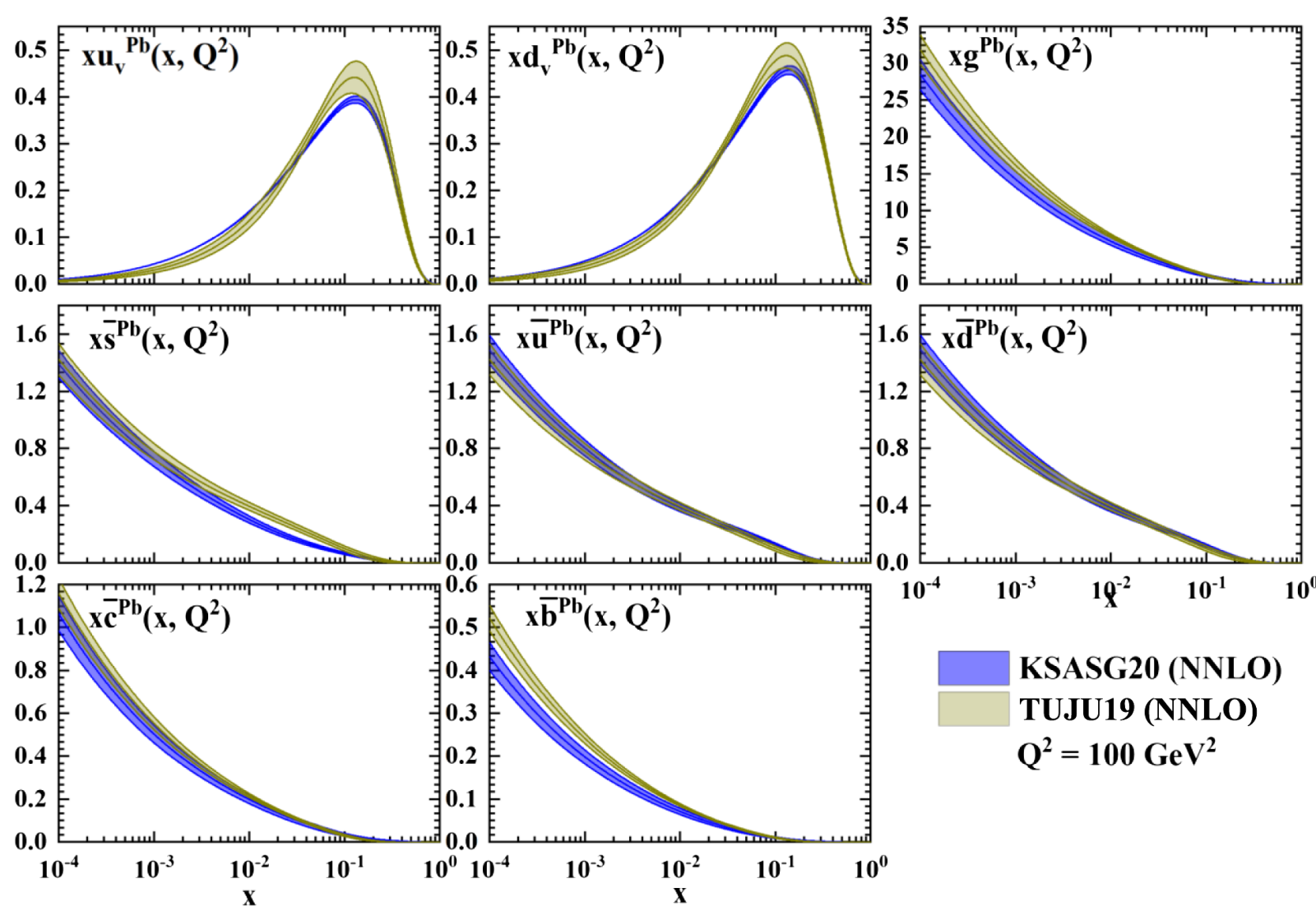

KSASG20 (NNLO)

TUJU19 (NNLO)

$\mathrm{Q}^{2}=100 \mathrm{GeV}^{2}$

FIG. 11. The nuclear PDFs at the scale $Q^{2}=100 \mathrm{GeV}^{2}$ for lead at NNLO accuracy, compared with the recent results from TUJU19 [4].
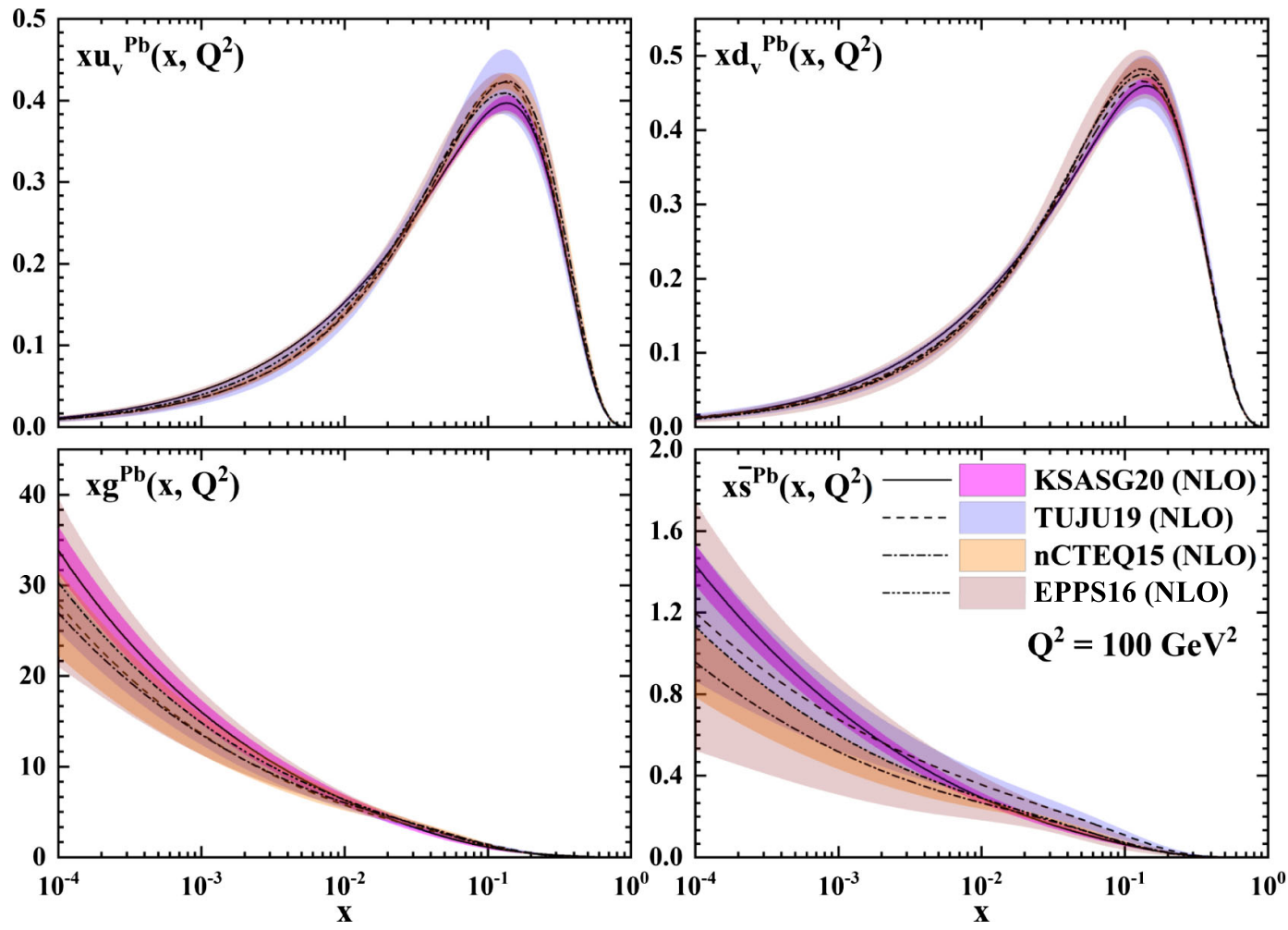

FIG. 12. The nuclear PDFs at the scale $Q^{2}=100 \mathrm{GeV}^{2}$ for lead at NLO accuracy, compared with results from $\mathrm{nCTEQ15}$ [18], EPPS16 [5], and TUJU19 [4]. The comparison is presented for the parton flavors $u_{v}, d_{v}, g$, and $\bar{s}$. 


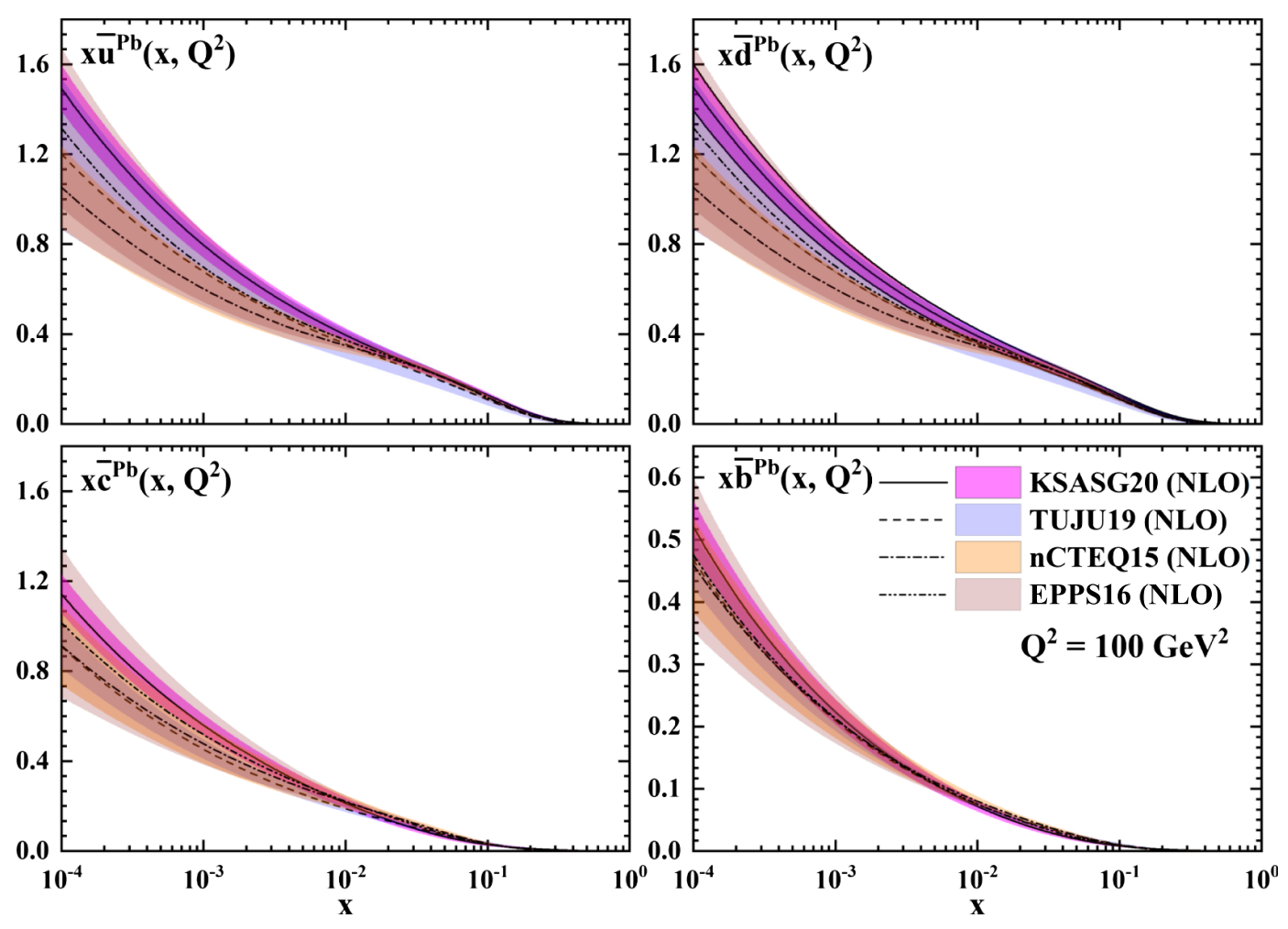

FIG. 13. Same as Fig. 12, but for the $\bar{u}, \bar{d}, \bar{c}$, and $\bar{b}$ PDFs.

JR PDFs [116]. The EPPS16 analysis was the first study which used data from the LHC for $Z$ and $W^{ \pm}$boson [28-30] and dijet production [27] in proton-lead collisions. These collider data provide further constraints for the gluon nuclear modifications and for the flavor separation. In the nCTEQ15 analysis, the nuclear PDFs are parametrized by a polynomial functional form, in which all the $A$-dependence is encoded in the coefficients of the parametrization. nCTEQ15 assumed $s=\bar{s}$ and the strange quark density is related to $\bar{u}+\bar{d}$ by an additional $A$-dependent factor, $s=\bar{s}=(\kappa(A) / 2)(\bar{u}+\bar{d})$. The TUJU19 analysis considered flavor symmetry for the sea quark densities, $\bar{u}=\bar{d}=s=\bar{s}$, while in EPPS16 and KSASG20 only $s=\bar{s}$ was used as a constraint.

After presenting the main properties of these recent nuclear PDFs, we compare their results at NLO accuracy. As can be seen from Fig. 12, for the $x u_{v}$ and $x d_{v}$ densities the KSASG20 results are in agreement in size with TUJU19, nCTEQ15, and EPPS16, and are well within their uncertainties, despite differences in the dataset and parametrization, etc. For the valence quark densities we find that both KSASG20 $x u_{v}$ and $x d_{v}$ slightly tend to stay below all other results at medium values of $x \sim 0.1$. Moderate differences for the gluon density and significant differences for the sea quark densities are observed. For both cases, EPPS16 exhibits relatively wider error bands compared with other analyses. The gluon distributions from nCTEQ15 and EPPS16 fall below the one of KSASG20.
In Fig. 13 we show a comparison for the sea quark distributions (upper row) and the charm and the bottom distributions (lower row). The latter two are perturbatively generated. Moderate differences between KSASG20, nCTEQ15 TUJU19, and EPPS16 can be seen. We find that the agreement between the results of KSASG2O and EPPS16 for the central values of $x \bar{u}, x \bar{d}$, and $x \bar{c}$ for the whole range of $x$ is slightly better than with the other two groups. The resulting uncertainties for KSASG20 are somewhat smaller. We should stress again that all results presented here are based on the choice $\Delta \chi^{2}=20$ for the tolerance. Choosing the larger tolerance value $\Delta \chi^{2}=50$, as preferred by other groups, the error bands of our nuclear PDFs would increase by a factor of $\sim 1.5$.

\section{Fit quality and comparison of data and theory}

In Tables I, II, III, V, IV, VI, and VII presented above in Sec. III, we have shown the values of $\chi^{2}$ per data point for each individual dataset, both for the NLO and the NNLO fits.

In total, we find for the KSASG20 fit at NLO that $\chi^{2}=4582$ with 4353 data points. With 18 free parameters, this leads to a $\chi^{2} /$ d.o.f $=1.05$ which indicates a relatively good fit. At NNLO, the KSASG20 fit leads to $\chi^{2} /$ d.o.f $=$ 1.04. This is only a moderate improvement upon inclusion of the higher-order QCD corrections.

While most datasets for nuclear and neutrino DIS experiments and Drell-Yan data satisfy the goodness of 
fit criterion, there are some experiments which stand out as having a poor fit. We also notice that for some datasets, $\chi^{2}$ is poor even for the NNLO fit. In addition, for some individual datasets $\chi^{2}$ increases as higher-order QCD corrections are included. A similar observation was made in previous analyses by TUJU19 [4] and nNNPDF1. 0 [1].

As one can see from Table $\mathrm{V}$, with the exception of $\nu \mathrm{Pb}$ data from CHORUS, for all other (anti)neutrino-nucleus DIS data the inclusion of higher-order QCD corrections leads to a better fit quality.

In order to illustrate our discussion, we present selected comparisons of the datasets used in this study to the corresponding NNLO theoretical predictions obtained using the KSASG2 0 NNLO nuclear PDFs. In the following plots, we combine experimental data for each nucleus for a wider range of $Q^{2}$ values (roughly between 1.8 and $67 \mathrm{GeV}^{2}$ ) where scaling violations are observed to cancel in the considered ratio. Our NNLO results have been calculated at $Q^{2}=5 \mathrm{GeV}^{2}$. In Fig. 14 such a comparison is displayed for the nuclear DIS data for the ratio $F_{2}^{A}\left(x, Q^{2}\right) / F_{2}^{C}\left(x, Q^{2}\right)$ as a function of $x$. In Fig. 15 we also compare our NNLO results for $F_{2}^{A}\left(x, Q^{2}\right) / F_{2}^{D}\left(x, Q^{2}\right)$ and $F_{2}^{A}\left(x, Q^{2}\right) / F_{2}^{L i}\left(x, Q^{2}\right)$ as a function of $x$ with some selected nuclear DIS data. The data shown in these plots are measured by the NMC and E139 Collaborations. The bands show the $68 \%$ uncertainty estimates with $\Delta \chi^{2}=20$. The comparisons presented in these plots demonstrate that the agreement between our NNLO theoretical predictions and the nuclear DIS experimental measurements varies between different data for different nuclei. Apart from a few data points in the small- $x$ region, the agreement with most of the data published by the NMC and E139 Collaborations is excellent.

A detailed comparison with the CHORUS data on neutrino(antineutrino) lead collisions [89] and the CDHSW data on neutrino(antineutrino) iron collisions [88] are shown in Figs. 16 and 17, respectively. The results are shown as a function of $Q^{2}$ for some selected bins of $x$ and $y$. The incident beam of neutrino(antineutrino) energies are not high enough to reach small values of $x$. Here we consider the range from $x=0.125$ to $x=0.65$, corresponding to the range between $y=0.3$ and $y=0.5$. Very good agreement is achieved for the neutrino(antineutrino)nucleus data presented in these plots for the whole region of $x$ and $Q^{2}$.

The data from the JLab experiment [45] included in this study provides important additional constraints on the shape and the uncertainty bands of nuclear PDFs in the high- $x$ and low- $\mathrm{Q}^{2}$ regime [6]. In order to show the agreement between our results using the extracted nuclear PDFs, in Fig. 18 we present the data/theory comparison for carbon $(\mathrm{C})$, aluminum $(\mathrm{Al})$, iron $(\mathrm{Fe})$, and lead $(\mathrm{Pb})$ nuclei. The bands in this plot show the $68 \%$ uncertainty estimates with $\Delta \chi^{2}=20$. We have again combined experimental data
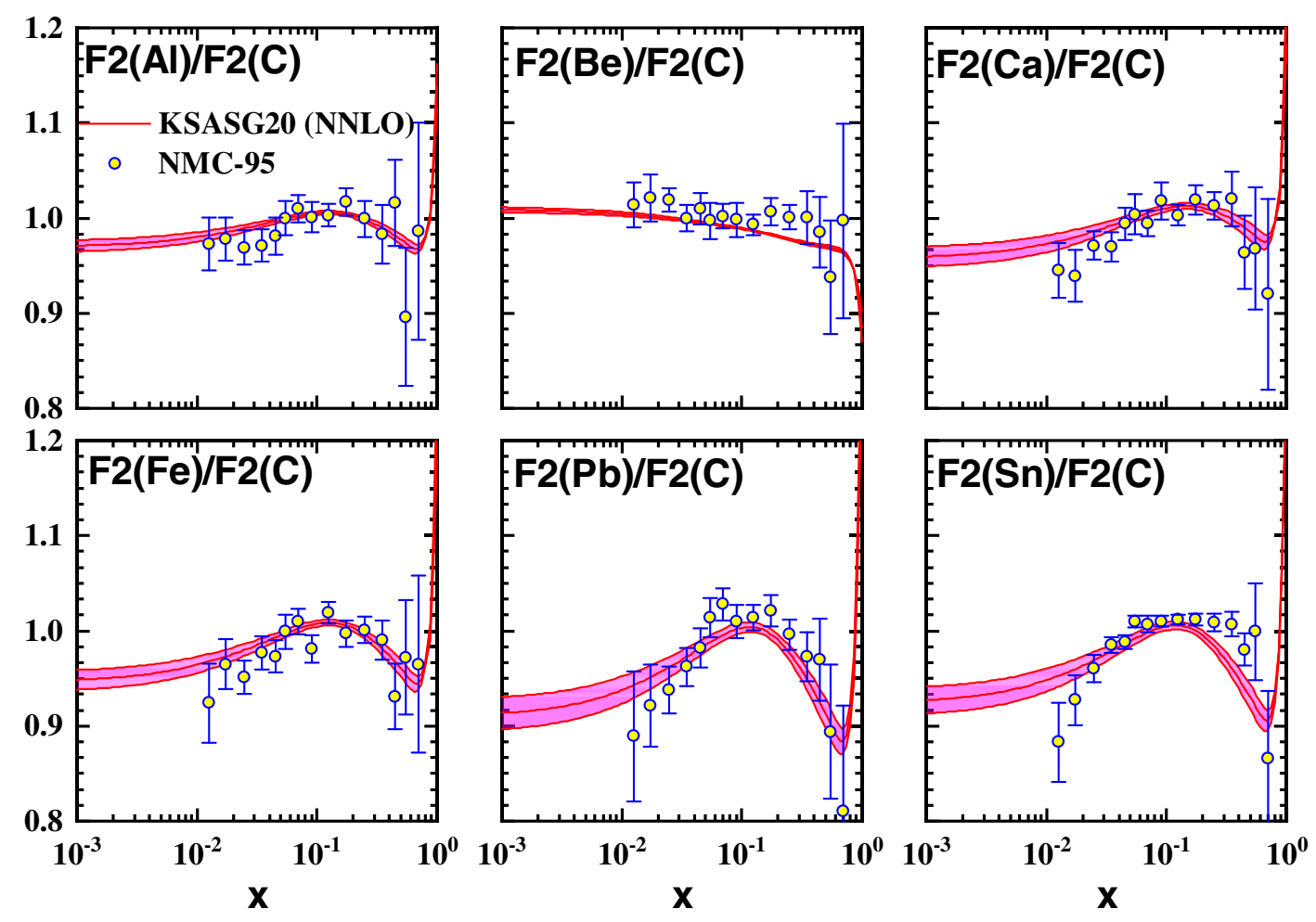

FIG. 14. Comparison of our NNLO results for the ratio $F_{2}^{A}\left(x, Q^{2}\right) / F_{2}^{C}\left(x, Q^{2}\right)$ as a function of $x$ with some selected nuclear DIS data from NMC-95. Our NNLO results have been calculated at $\mathrm{Q}^{2}=5 \mathrm{GeV}^{2}$. The bands show the $68 \%$ uncertainty estimate with $\Delta \chi^{2}=20$. 

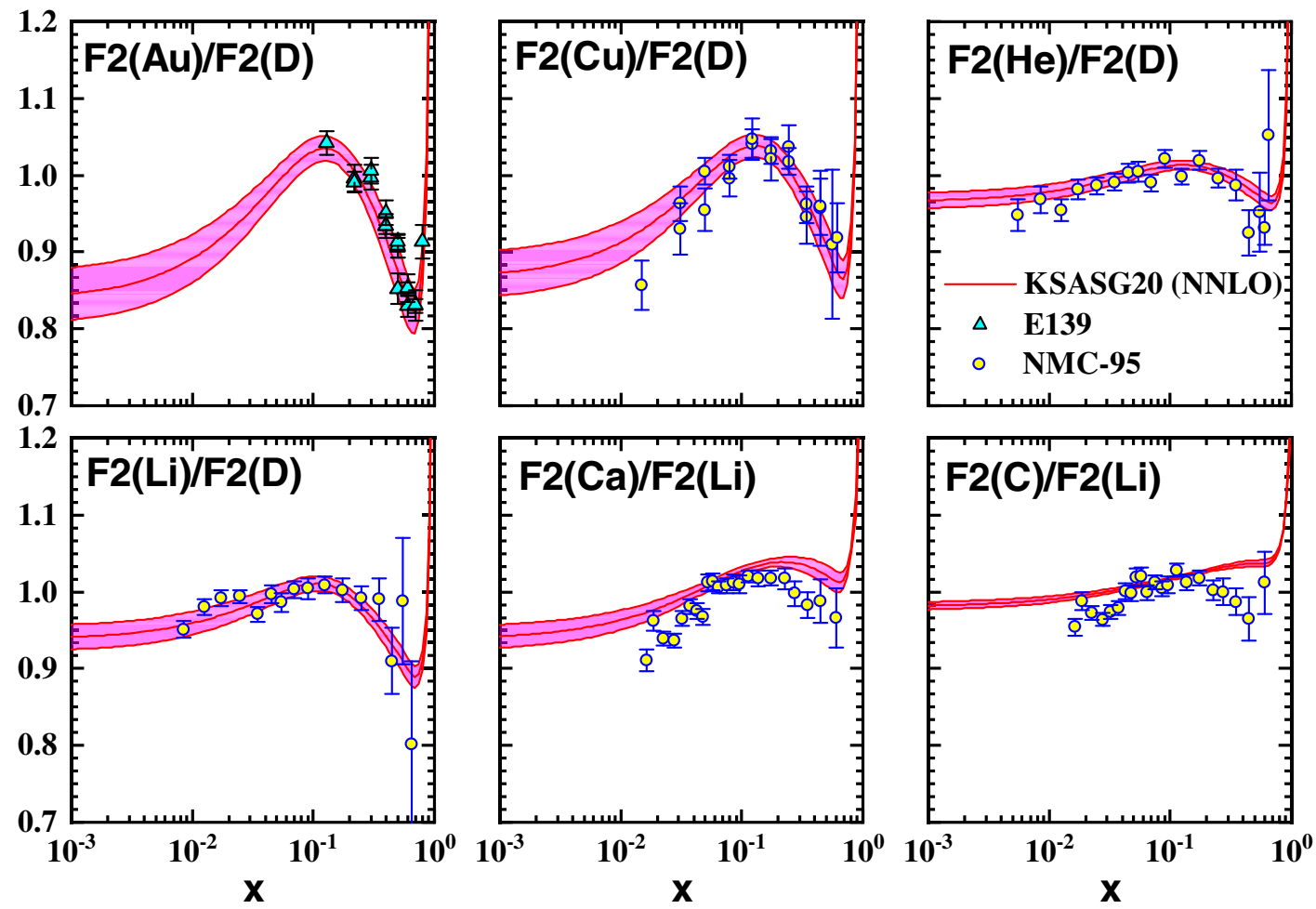

FIG. 15. Comparison of our NNLO results calculated at $Q^{2}=5 \mathrm{GeV}^{2}$ for the ratios $F_{2}^{A}\left(x, Q^{2}\right) / F_{2}^{D}\left(x, Q^{2}\right)$ and $F_{2}^{A}\left(x, Q^{2}\right) / F_{2}^{L i}\left(x, Q^{2}\right)$ as a function of $x$ with some selected nuclear DIS data from E139 and NMC-95.
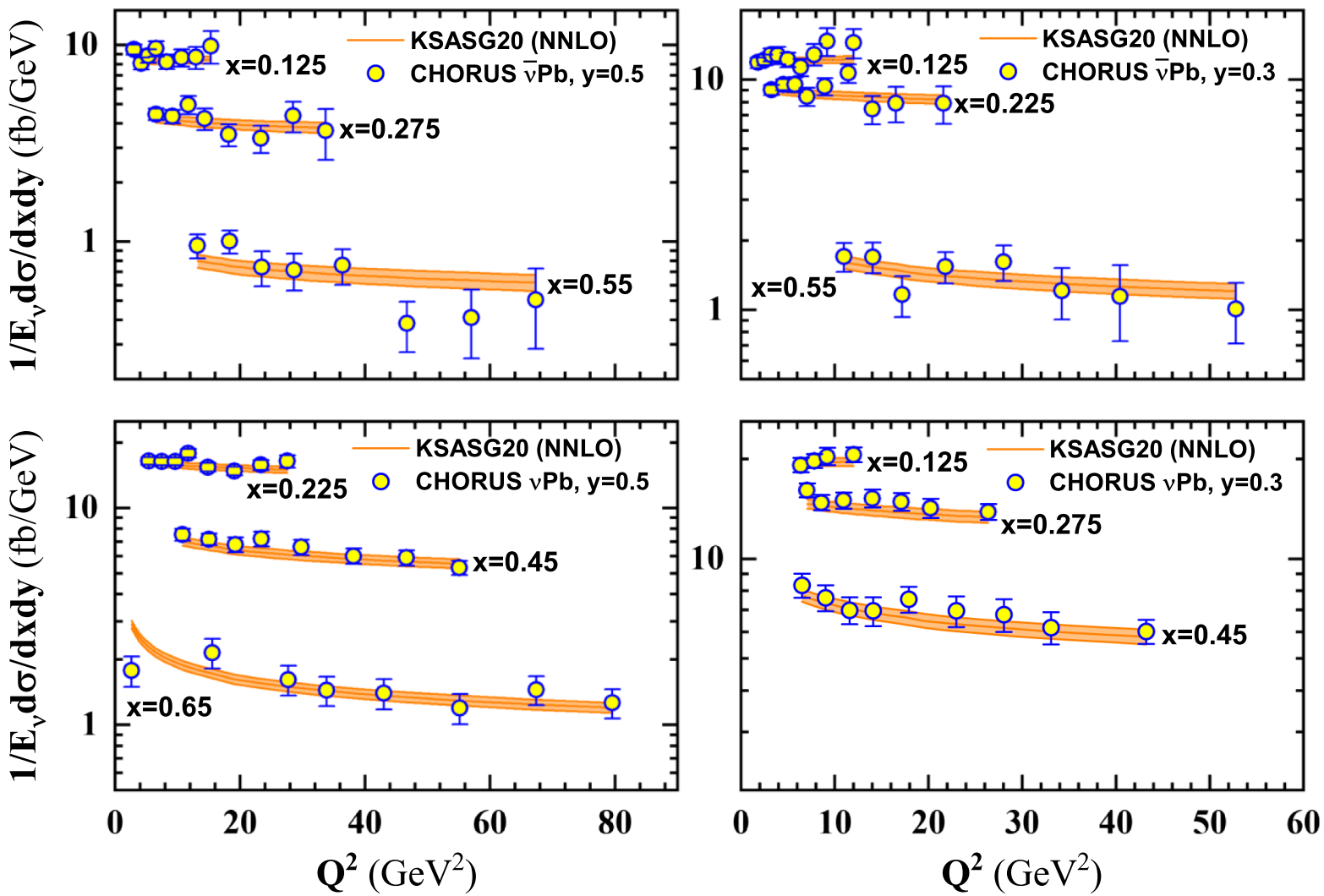

FIG. 16. The neutrino(antineutrino) lead DIS data from the CHORUS measurements [89], compared with the KSASG20 NNLO results. The bands show the $68 \%$ uncertainty estimates with $\Delta \chi^{2}=20$. 

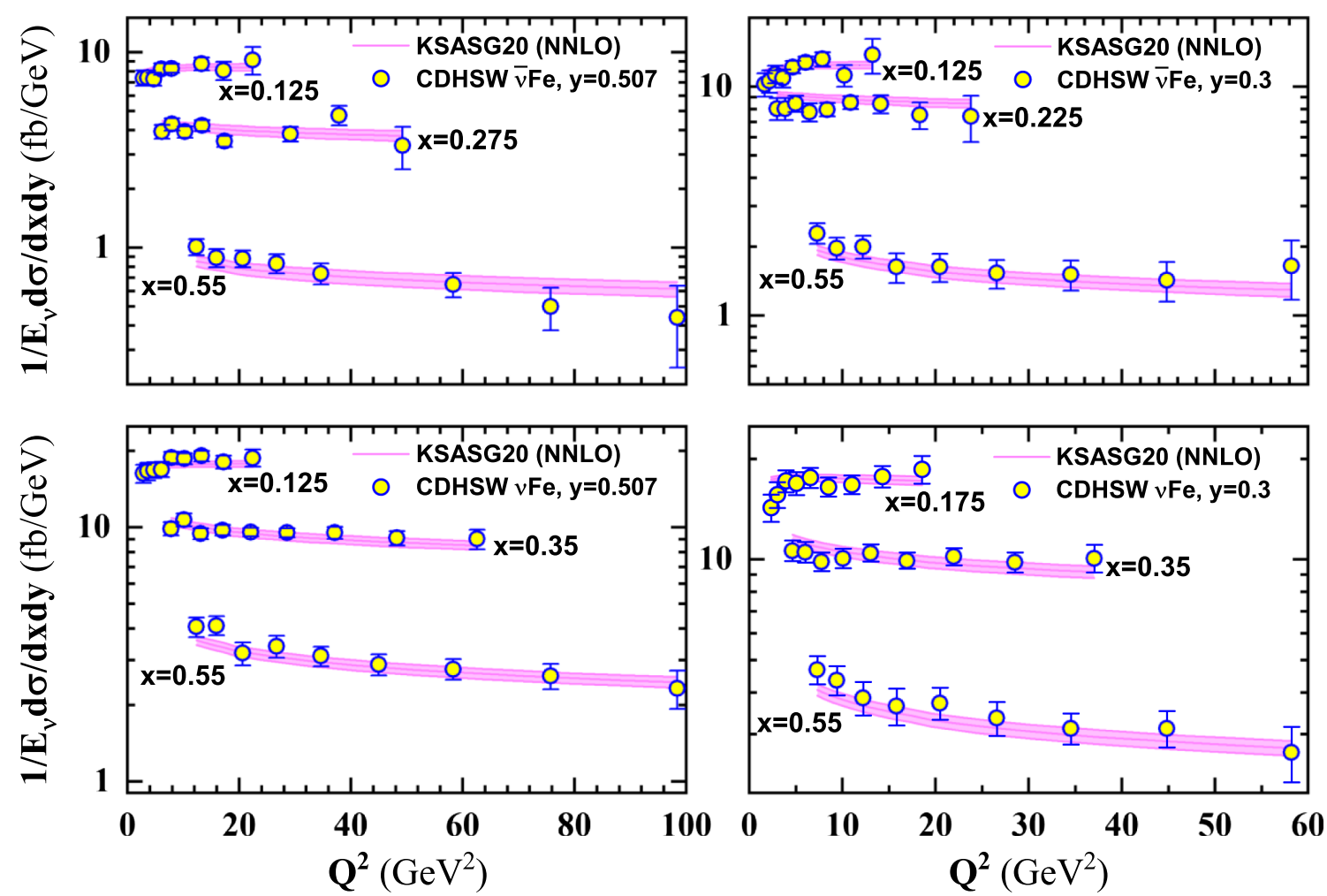

FIG. 17. Same as Fig. 16, but for the neutrino(antineutrino) iron DIS data from the CDHSW measurements [88].
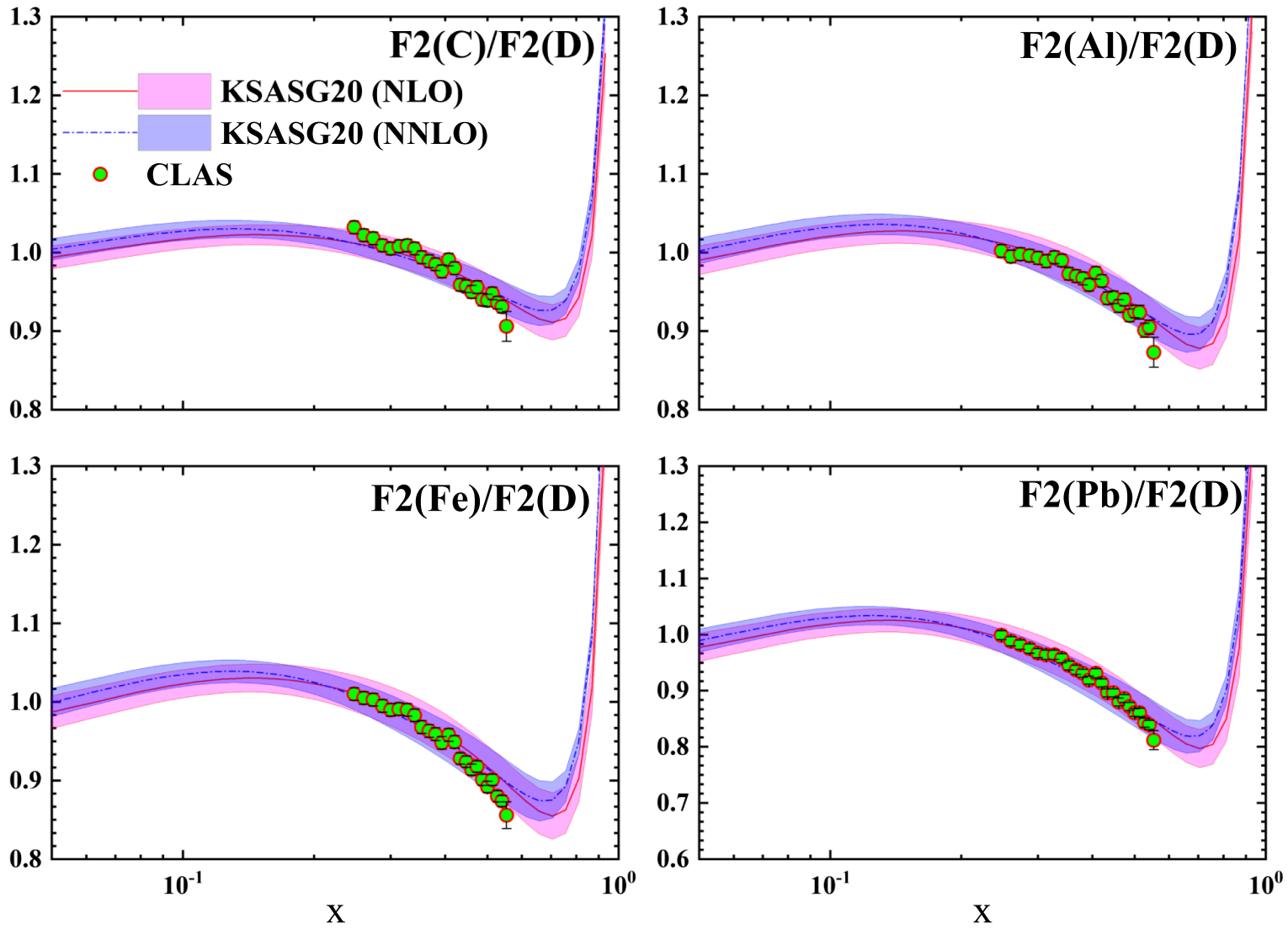

FIG. 18. Comparison of our NLO and NNLO results calculated at $Q^{2}=3 \mathrm{GeV}^{2}$ for the structure function ratios $F_{2}^{A}\left(x, Q^{2}\right) / F_{2}^{D}\left(x, Q^{2}\right)$ with the data from Jefferson Lab CLAS [45] as a function of $x$. 


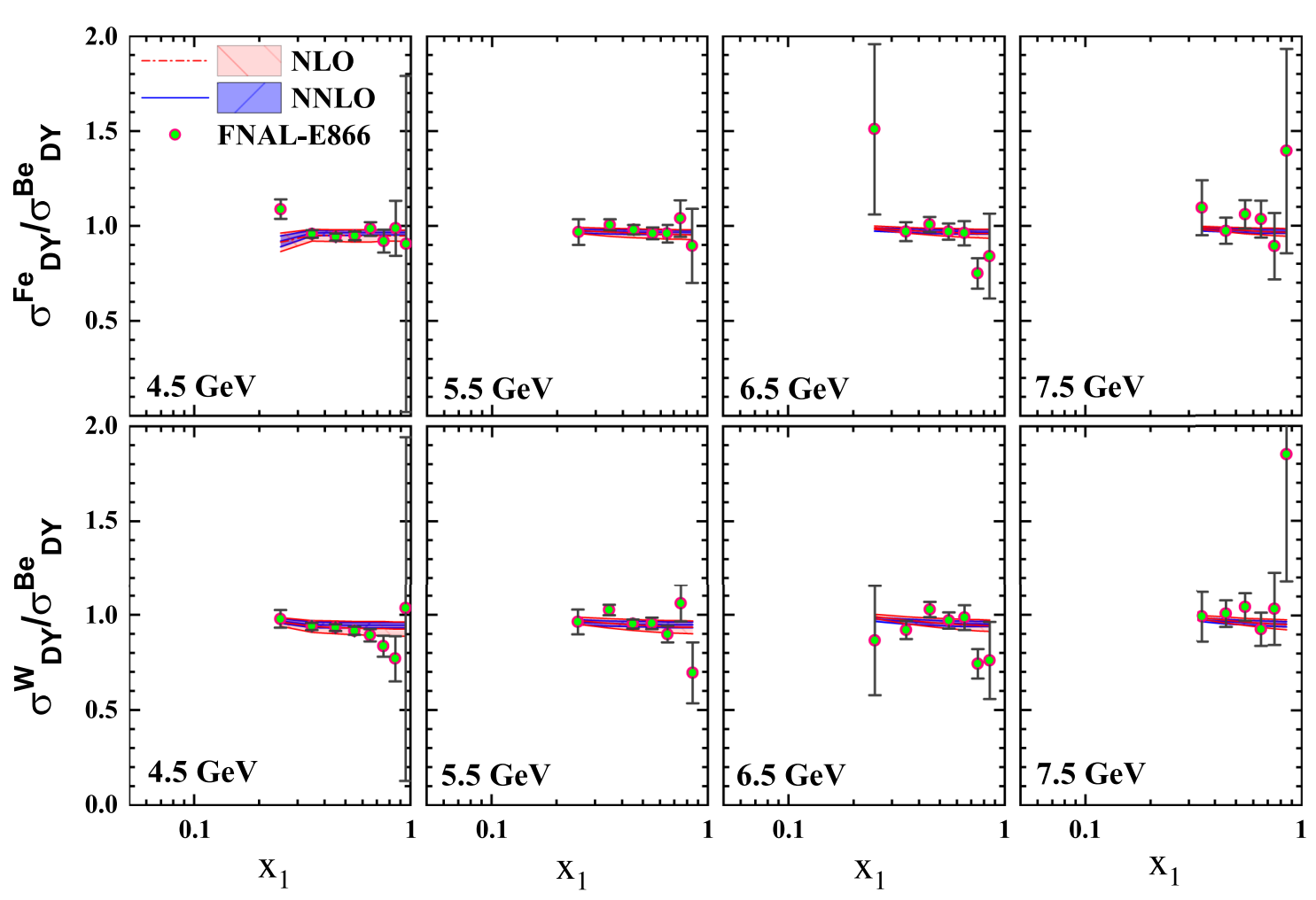

FIG. 19. Comparison of the KSASG2 0 NLO and NNLO results along with their uncertainties for the Drell-Yan cross section ratios $\sigma_{\mathrm{DY}}^{A} / \sigma_{\mathrm{DY}}^{A^{\prime}}$ with data for some selected nuclear targets from the Fermilab experiment E866 [104].

for different $Q^{2}$-values (between 1.8 and $46 \mathrm{GeV}^{2}$ ) while our theory results are calculated at $Q^{2}=3 \mathrm{GeV}^{2}$. As one can see, our NLO and NNLO theory results nicely describe the recent JLab CLAS [45] data.

We now turn to the Drell-Yan cross section ratios $\sigma_{\mathrm{DY}}^{A} / \sigma_{\mathrm{DY}}^{A^{\prime}}$. In Fig. 19, we display the cross section ratio measured by the Fermilab experiment E866 for some selected nuclear targets [104]. Our NLO and NNLO results along with their uncertainties are shown as well. The DrellYan cross sections are presented in four bins of the invariant mass and are shown as a function of the proton momentum fraction $x_{1}$. As one can see, except for some isolated points with relatively large errors, the KSASG20 NLO theory results describe the data well. In addition, the results show that the uncertainty bands at NNLO are slightly smaller than at NLO.

\section{SUMMARY AND CONCLUSIONS}

In summary, in this work we have introduced a new set of nuclear PDFs at NLO and NNLO accuracy in pQCD. KSASG2 0 nuclear PDFs are obtained from most up-to-date experimental data, including neutral-current nuclear DIS with several nuclear targets, charged-current neutrino DIS, and Drell-Yan cross section measurements. The combination of these data are sensitive to the flavor decomposition of nuclei. Our analysis also incorporates the most recent DIS data from the Jefferson Lab CLAS and Hall C experiments. For these specific data sets, we take into account target mass corrections and higher twist effects which are mainly important in the region of large $x$ and intermediate-to-low $\mathrm{Q}^{2}$. Heavy-quark mass effects are included in the FONLL general-mass variable-flavornumber scheme for charm and bottom quarks.

Our determination of nuclear PDFs includes error estimates obtained within the Hessian method with the tolerance criterion of $\Delta \chi^{2}=20$. The effects arising from the inclusion of higher-order QCD corrections are investigated. We found only small differences between the NLO and NNLO QCD fits, both for the shape and size of the uncertainty bands. The inclusion of higher-order QCD corrections slightly improves the description of the nuclear data analyzed in this study, but there is no strong indication that NNLO corrections are required by the data. The largest differences appear for the gluon nuclear PDF, where our NNLO fit leads to a slight decrease in the uncertainties.

In contrast to other recent analyses, such as nNNPDF 2 . 0 [1] and nNNPDF2.1 [2], which use Monte Carlo techniques based on the NNPDF framework, and TUJU19 [4], which is based on the CTEQ framework, we parametrize the nuclear PDFs considering nuclear correction factors. To this end, a modern set of parton distribution functions for free protons, namely CT18, is considered as reference. Our results are consistent, within uncertainties, with the previous determinations of nuclear PDFs available in the 
literature, in particular for nCTEQ15 [18], EPPS16 [5], and TUJU19, which are based on a different selection of data sets and assumptions. However, we found a number of differences which only occur in regions without any constraints from data. These differences can be attributed to different assumptions such as the input parametrization of the nuclear modification factors.

The nuclear PDF analysis presented in this article represents the first step of a broader study. A number of improvements are foreseen for the near future. While the data used in the present study allowed us to determine the nuclear quark and antiquark densities, the nuclear gluon PDF is only loosely constrained. This is actually the most important limitation of the KSASG2 0 nuclear PDF analysis. To resolve this limitation, we plan to include additional datasets, especially from present and future measurements of proton-lead and lead-lead collisions at the CERN-LHC, which are expected to provide direct information on the nuclear gluon modifications and more stringent flavordependence constraints. These include, for example, the data from PHENIX and STAR for inclusive pion production in deuteron-gold $(\mathrm{d}-\mathrm{Au})$ collisions $[117,118]$, neutral pion production data from PHENIX [117], and the charged and neutral pion data from the STAR experiment $[118,119]$. Also data from $\mathrm{p}-\mathrm{Pb}$ collisions from the ATLAS and CMS Collaborations at the LHC, which have been included in the analyses of nuclear PDFs performed in Refs. [2,5], are expected to improve the constraints of the nuclear gluon density at large momentum fractions. With more precise data, it might be necessary in the future to consider more flexible parametrizations.

We can also expect that the electron-ion collider [120], the Large Hadron-Electron Collider [121], or a Future Circular Collider $[122,123]$ could provide precise data for nuclear PDF analyses. It would be very interesting to repeat the analysis described here using additional observables from hadron colliders such as the LHC.

The NLO and NNLO nuclear PDF sets presented in this work are available in the LHAPDF format [114] for all relevant nuclei from $A=2$ to $A=208$, and can be obtained from the authors upon request.

\section{ACKNOWLEDGMENTS}

We are thankful to Petja Paakkinen, Hamed Abdolmaleki, Marina Walt, Ilkka Helenius, and Muhammad Goharipour for many helpful discussions and comments. We also gratefully thank Valerio Bertone for a discussion regarding subtle differences between various heavy quark mass schemes and their numerical implementation. The authors thank the School of Particles and Accelerators, Institute for Research in Fundamental Sciences (IPM) for financial support of this project. Hamzeh Khanpour also is grateful to the University of Science and Technology of Mazandaran for financial support of this project.
[1] R. A. Khalek, J. J. Ethier, and J. Rojo (NNPDF Collaboration), Nuclear parton distributions from lepton-nucleus scattering and the impact of an electron-ion collider, Eur. Phys. J. C 79, 471 (2019).

[2] R. A. Khalek, J. J. Ethier, J. Rojo, and G. van Weelden, nNNPDF2.0: Quark flavor separation in nuclei from LHC data, J. High Energy Phys. 09 (2020) 183.

[3] H. Khanpour and S. Atashbar Tehrani, Global analysis of nuclear parton distribution functions and their uncertainties at next-to-next-to-leading order, Phys. Rev. D 93, 014026 (2016).

[4] M. Walt, I. Helenius, and W. Vogelsang, Open-source QCD analysis of nuclear parton distribution functions at NLO and NNLO, Phys. Rev. D 100, 096015 (2019).

[5] K. J. Eskola, P. Paakkinen, H. Paukkunen, and C. A. Salgado, EPPS16: Nuclear parton distributions with LHC data, Eur. Phys. J. C 77, 163 (2017).

[6] H. Paukkunen and P. Zurita, Can we fit nuclear PDFs with the high-x CLAS data?, Eur. Phys. J. C 80, 381 (2020).

[7] P. Ru, S. A. Kulagin, R. Petti, and B. W. Zhang, Study of $W^{ \pm}$and $Z$ boson production in proton-lead collisions at the LHC with Kulagin-Petti nuclear parton distributions, Phys. Rev. D 94, 113013 (2016).
[8] R. Wang, X. Chen, and Q. Fu, Global study of nuclear modifications on parton distribution functions, Nucl. Phys. B920, 1 (2017).

[9] A. Kusina, F. Lyonnet, D. B. Clark, E. Godat, T. Ježo, K. Kovařík, F. I. Olness, I. Schienbein, and J. Y. Yu, Vector boson production in $\mathrm{pPb}$ and $\mathrm{PbPb}$ collisions at the $\mathrm{LHC}$ and its impact on nCTEQ15 PDFs, Eur. Phys. J. C 77, 488 (2017).

[10] S. Atashbar Tehrani, Nuclear parton densities and their uncertainties at the next-to-leading order, Phys. Rev. C 86, 064301 (2012).

[11] S. A. Kulagin and R. Petti, Nuclear parton distributions and the Drell-Yan process, Phys. Rev. C 90, 045204 (2014).

[12] D. de Florian, R. Sassot, P. Zurita, and M. Stratmann, Global analysis of nuclear parton distributions, Phys. Rev. D 85, 074028 (2012).

[13] K. J. Eskola, H. Paukkunen, and C. A. Salgado, An improved global analysis of nuclear parton distribution functions including RHIC data, J. High Energy Phys. 07 (2008) 102.

[14] K. Kovarik, I. Schienbein, F. I. Olness, J. Y. Yu, C. Keppel, J. G. Morfin, J. F. Owens, and T. Stavreva, Nuclear Corrections in Neutrino-Nucleus DIS and Their 
Compatibility with Global NPDF Analyses, Phys. Rev. Lett. 106, 122301 (2011).

[15] K. J. Eskola, H. Paukkunen, and C. A. Salgado, EPS09: A new generation of NLO and LO nuclear parton distribution functions, J. High Energy Phys. 04 (2009) 065.

[16] M. Hirai, S. Kumano, and M. Miyama, Determination of nuclear parton distributions, Phys. Rev. D 64, 034003 (2001).

[17] M. Hirai, S. Kumano, and T.-H. Nagai, Determination of nuclear parton distribution functions and their uncertainties in next-to-leading order, Phys. Rev. C 76, 065207 (2007).

[18] K. Kovarik et al., nCTEQ15-Global analysis of nuclear parton distributions with uncertainties in the CTEQ framework, Phys. Rev. D 93, 085037 (2016).

[19] D. de Florian, R. Sassot, M. Stratmann, and P. Zurita, Global analysis of nuclear PDFs, arXiv:1204.3797.

[20] Y.L. Dokshitzer, Calculation of the structure functions for deep inelastic scattering and e+ e- annihilation by perturbation theory in quantum chromodynamics, Zh. Eksp. Teor. Fiz. 73, 1216 (1977) [Sov. Phys. JETP 46, 641 (1977)].

[21] V. N. Gribov and L. N. Lipatov, Deep inelastic e p scattering in perturbation theory, Yad. Fiz. 15, 781 (1972) [Sov. J. Nucl. Phys. 15, 438 (1972)].

[22] L. N. Lipatov, The parton model and perturbation theory, Sov. J. Nucl. Phys. 20, 94 (1975).

[23] G. Altarelli and G. Parisi, Asymptotic freedom in parton language, Nucl. Phys. B126, 298 (1977).

[24] S. Alekhin et al., HERAFitter, Eur. Phys. J. C 75, 304 (2015).

[25] V. Bertone, S. Carrazza, and J. Rojo, APFEL: A PDF evolution library with QED corrections, Comput. Phys. Commun. 185, 1647 (2014).

[26] T. J. Hou et al., New CTEQ global analysis of quantum chromodynamics with high-precision data from the LHC, Phys. Rev. D 103, 014013 (2021).

[27] S. Chatrchyan et al. (CMS Collaboration), Studies of dijet transverse momentum balance and pseudorapidity distributions in $\mathrm{pPb}$ collisions at $\sqrt{s_{\mathrm{NN}}}=5.02 \mathrm{TeV}$, Eur. Phys. J. C 74, 2951 (2014).

[28] V. Khachatryan et al. (CMS Collaboration), Study of W boson production in $\mathrm{pPb}$ collisions at $\sqrt{s_{\mathrm{NN}}}=5.02 \mathrm{TeV}$, Phys. Lett. B 750, 565 (2015).

[29] V. Khachatryan et al. (CMS Collaboration), Study of Z boson production in $\mathrm{pPb}$ collisions at $\sqrt{s_{N N}}=5.02 \mathrm{TeV}$, Phys. Lett. B 759, 36 (2016).

[30] G. Aad et al. (ATLAS Collaboration), $Z$ boson production in $p+\mathrm{Pb}$ collisions at $\sqrt{s_{N N}}=5.02 \mathrm{TeV}$ measured with the ATLAS detector, Phys. Rev. C 92, 044915 (2015).

[31] A. M. Sirunyan et al. (CMS Collaboration), Observation of nuclear modifications in $W^{ \pm}$boson production in $\mathrm{pPb}$ collisions at $\sqrt{s_{\mathrm{NN}}}=8.16 \mathrm{TeV}$, Phys. Lett. B 800, 135048 (2020).

[32] N. Armesto, H. Paukkunen, J. M. Penín, C. A. Salgado, and P. Zurita, An analysis of the impact of LHC Run I proton-lead data on nuclear parton densities, Eur. Phys. J. C 76, 218 (2016).

[33] K. J. Eskola, P. Paakkinen, and H. Paukkunen, Non-quadratic improved Hessian PDF reweighting and application to CMS dijet measurements at $5.02 \mathrm{TeV}$, Eur. Phys. J. C 79, 511 (2019).

[34] H. Paukkunen and C. A. Salgado, Agreement of Neutrino Deep Inelastic Scattering Data with Global Fits of Parton Distributions, Phys. Rev. Lett. 110, 212301 (2013).

[35] H. Paukkunen and P. Zurita, PDF reweighting in the Hessian matrix approach, J. High Energy Phys. 12 (2014) 100.

[36] K. J. Eskola, I. Helenius, P. Paakkinen, and H. Paukkunen, A QCD analysis of LHCb D-meson data in $\mathrm{p}+\mathrm{Pb}$ collisions, J. High Energy Phys. 05 (2020) 037.

[37] R. Aaij et al. (LHCb Collaboration), Study of prompt $\mathrm{D}^{0}$ meson production in $p \mathrm{~Pb}$ collisions at $\sqrt{s_{\mathrm{NN}}}=5 \mathrm{TeV}$, J. High Energy Phys. 10 (2017) 090.

[38] H. Paukkunen, Nuclear PDFs in the beginning of the LHC era, Nucl. Phys. A926, 24 (2014).

[39] K. Rith, Present status of the EMC effect, Subnuclear series 51, 431 (2015).

[40] K. J. Eskola, Global analysis of nuclear PDFs-latest developments, Nucl. Phys. A910, 163 (2013).

[41] I. Sick and D. Day, The EMC effect of nuclear matter, Phys. Lett. B 274, 16 (1992).

[42] N. Fomin et al., Scaling of the $F_{2}$ Structure Function in Nuclei and Quark Distributions at $x>1$, Phys. Rev. Lett. 105, 212502 (2010).

[43] I. Niculescu, J. Arrington, R. Ent, and C. E. Keppel, Moments of nuclear and nucleon structure functions at low $Q^{2}$ and the momentum sum rule, Phys. Rev. C 73, 045206 (2006).

[44] J. Seely et al., New Measurements of the EMC Effect in Very Light Nuclei, Phys. Rev. Lett. 103, 202301 (2009).

[45] B. Schmookler et al. (CLAS Collaboration), Modified structure of protons and neutrons in correlated pairs, Nature (London) 566, 354 (2019).

[46] I. Schienbein, V. A. Radescu, G. P. Zeller, M. E. Christy, C. E. Keppel, K. S. McFarland, W. Melnitchouk, F. I. Olness, M. H. Reno, F. Steffens et al., A review of target mass corrections, J. Phys. G 35, 053101 (2008).

[47] H. Georgi and H. D. Politzer, Freedom at moderate energies: Masses in color dynamics, Phys. Rev. D 14, 1829 (1976).

[48] V. Bertone, Higher order and heavy Quark mass effects in the determination of parton distribution functions, http:// nnpdf.mi.infn.it/wp-content/uploads/2017/10/VBertonethesis.pdf.

[49] M. Goharipour and S. Rostami, Implementation of target mass corrections and higher-twist effects in the xFitter framework, Phys. Rev. D 101, 074015 (2020).

[50] F. M. Steffens, M. D. Brown, W. Melnitchouk, and S. Sanches, Parton distributions in the presence of target mass corrections, Phys. Rev. C 86, 065208 (2012).

[51] E. Moffat, T. C. Rogers, W. Melnitchouk, N. Sato, and F. Steffens, What does kinematical target mass sensitivity in DIS reveal about hadron structure?, Phys. Rev. D 99, 096008 (2019).

[52] A. Accardi, L. T. Brady, W. Melnitchouk, J. F. Owens, and N. Sato, Constraints on large- $x$ parton distributions from new weak boson production and deep-inelastic scattering data, Phys. Rev. D 93, 114017 (2016). 
[53] H. Khanpour, S. T. Monfared, and S. Atashbar Tehrani, Nucleon spin structure functions at NNLO in the presence of target mass corrections and higher twist effects, Phys. Rev. D 95, 074006 (2017).

[54] O. Nachtmann, Positivity constraints for anomalous dimensions, Nucl. Phys. B63, 237 (1973).

[55] J. Blumlein, The theory of deeply inelastic scattering, Prog. Part. Nucl. Phys. 69, 28 (2013).

[56] A. Accardi, M. E. Christy, C. E. Keppel, W. Melnitchouk, P. Monaghan, J. G. Morfín, and J. F. Owens, New parton distributions from large- $x$ and low- $Q^{2}$ data, Phys. Rev. D 81, 034016 (2010).

[57] L. T. Brady, A. Accardi, T. J. Hobbs, and W. Melnitchouk, Next-to leading order analysis of target mass corrections to structure functions and asymmetries, Phys. Rev. D 84, 074008 (2011); , Erratum, Phys. Rev. D 85, 039902 (2012).

[58] J.w. Qiu and G. F. Sterman, QCD and rescattering in nuclear targets, Int. J. Mod. Phys. E 12, 149 (2003).

[59] A. Accardi et al., A critical appraisal and evaluation of modern PDFs, Eur. Phys. J. C 76, 471 (2016).

[60] A. D. Martin, W. J. Stirling, R. S. Thorne, and G. Watt, Parton distributions for the LHC, Eur. Phys. J. C 63, 189 (2009).

[61] M. Cacciari, M. Greco, and P. Nason, The P(T) spectrum in heavy flavor hadroproduction, J. High Energy Phys. 05 (1998) 007.

[62] S. Forte, E. Laenen, P. Nason, and J. Rojo, Heavy quarks in deep-inelastic scattering, Nucl. Phys. B834, 116 (2010).

[63] M. Krämer, F. I. Olness, and D. E. Soper, Treatment of heavy quarks in deeply inelastic scattering, Phys. Rev. D 62, 096007 (2000).

[64] W. K. Tung, S. Kretzer, and C. Schmidt, Open heavy flavor production in QCD: Conceptual framework and implementation issues, J. Phys. G 28, 983 (2002).

[65] M. Tanabashi et al. (Particle Data Group Collaboration), Review of particle physics, Phys. Rev. D 98, 030001 (2018).

[66] J. Ashman et al. (European Muon Collaboration), A measurement of the ratio of the nucleon structure function in copper and deuterium, Z. Phys. C 57, 211 (1993).

[67] P. Amaudruz et al. (New Muon Collaboration), A reevaluation of the nuclear structure function ratios for $\mathrm{D}, \mathrm{He}$, Li-6, C and Ca, Nucl. Phys. B441, 3 (1995).

[68] J. Ashman et al. (European Muon Collaboration), Measurement of the ratios of deep inelastic Muon-Nucleus cross-sections on various nuclei compared to deuterium, Phys. Lett. B 202, 603 (1988).

[69] M. Arneodo et al. (New Muon Collaboration), The structure function ratios F2(Li) / F2(D) and F2(C) / F2(D) at small x, Nucl. Phys. B441, 12 (1995).

[70] G. Bari et al. (BCDMS Collaboration), A measurement of nuclear effects in deep inelastic Muon scattering on deuterium, nitrogen and iron targets, Phys. Lett. 163B, 282 (1985).

[71] M. Arneodo et al. (New Muon Collaboration), The a dependence of the nuclear structure function ratios, Nucl. Phys. B481, 3 (1996).
[72] A. C. Benvenuti et al. (BCDMS Collaboration), Nuclear effects in deep inelastic Muon scattering on deuterium and iron targets, Phys. Lett. B 189, 483 (1987).

[73] M. Arneodo et al. (New Muon Collaboration), The $Q^{2}$ dependence of the structure function ratio $F_{2}^{S n} / F_{2}^{C}$ and the difference $R_{S n}-R_{C}$ in deep inelastic muon scattering, Nucl. Phys. B481, 23 (1996).

[74] J. Gomez et al., Measurement of the A-dependence of deep inelastic electron scattering, Phys. Rev. D 49, 4348 (1994).

[75] A. Bodek et al., A Comparison of the Deep Inelastic Structure Functions of Deuterium and Aluminum Nuclei, Phys. Rev. Lett. 51, 534 (1983).

[76] A. Bodek et al., Electron Scattering from Nuclear Targets and Quark Distributions in Nuclei, Phys. Rev. Lett. 50, 1431 (1983).

[77] M. Arneodo et al. (European Muon Collaboration), Measurements of the nucleon structure function in the range $0.002<x<0.17$ and $0.2<Q^{2}<8 \mathrm{GeV}^{2}$ in deuterium, carbon and calcium, Nucl. Phys. B333, 1 (1990).

[78] S. Dasu et al., Measurement of the Difference in $\mathrm{R}=\sigma_{L} \sigma_{T}$ and of $\sigma^{A} / \sigma^{D}$ in Deep Inelastic e-D, e-Fe and e-Au Scattering, Phys. Rev. Lett. 60, 2591 (1988).

[79] K. Ackerstaff et al. (HERMES Collaboration), Nuclear effects on $\mathrm{R}=\sigma_{L} / \sigma_{T}$ in deep inelastic scattering, Phys. Lett. B 475, 386 (2000); , Erratum, Phys. Lett. B 567, 339 (2003).

[80] M. R. Adams et al. (E665 Collaboration), Saturation of Shadowing at Very Low $x_{\mathrm{BJ}}$, Phys. Rev. Lett. 68, 3266 (1992).

[81] M. R. Adams et al. (E665 Collaboration), Shadowing in inelastic scattering of muons on carbon, calcium and lead at low x(Bj), Z. Phys. C 67, 403 (1995).

[82] M. Arneodo et al. (New Muon Collaboration), Measurement of the proton and deuteron structure functions, F2(p) and F2(d), and of the ratio sigma-L / sigma-T, Nucl. Phys. B483, 3 (1997).

[83] M. R. Adams et al. (E665 Collaboration), Proton and deuteron structure functions in muon scattering at 470-GeV, Phys. Rev. D 54, 3006 (1996).

[84] A. C. Benvenuti et al. (BCDMS Collaboration), A high statistics measurement of the deuteron structure functions F2 $\left(\mathrm{X}, Q^{2}\right)$ and $\mathrm{R}$ from deep inelastic Muon scattering at high $Q^{2}$, Phys. Lett. B 237, 592 (1990).

[85] A. Airapetian et al. (HERMES Collaboration), Inclusive measurements of inelastic electron and positron scattering from unpolarized hydrogen and deuterium targets, J. High Energy Phys. 05 (2011) 126.

[86] M. Arneodo et al. (New Muon Collaboration), Accurate measurement of $F_{2}^{d} / F_{2}^{p}$ and $R^{d}-R^{p}$, Nucl. Phys. B487, 3 (1997).

[87] F. Gross, Making the case for Jefferson Lab, J. Phys. Conf. Ser. 299, 012001 (2011).

[88] J. P. Berge et al., A measurement of differential cross-sections and nucleon structure functions in charged current neutrino interactions on iron, Z. Phys. C 49, 187 (1991).

[89] G. Onengut et al. (CHORUS Collaboration), Measurement of nucleon structure functions in neutrino scattering, Phys. Lett. B 632, 65 (2006). 
[90] M. Tzanov et al. (NuTeV Collaboration), Precise measurement of neutrino and anti-neutrino differential cross sections, Phys. Rev. D 74, 012008 (2006).

[91] D. MacFarlane et al., Nucleon structure functions from high-energy neutrino interactions with iron and QCD results, Z. Phys. C 26, 1 (1984).

[92] I. Schienbein, J. Y. Yu, C. Keppel, J. G. Morfin, F. Olness, and J.F. Owens, Nuclear parton distribution functions from neutrino deep inelastic scattering, Phys. Rev. D 77, 054013 (2008).

[93] I. Schienbein, J. Y. Yu, K. Kovarik, C. Keppel, J. G. Morfin, F. Olness, and J. F. Owens, PDF nuclear corrections for charged and neutral current processes, Phys. Rev. D 80, 094004 (2009).

[94] S. M. M. Nejad, H. Khanpour, S. Atashbar Tehrani, and M. Mahdavi, QCD analysis of nucleon structure functions in deep-inelastic neutrino-nucleon scattering: Laplace transform and Jacobi polynomials approach, Phys. Rev. C 94, 045201 (2016).

[95] H. Paukkunen and C. A. Salgado, Compatibility of neutrino DIS data and global analyses of parton distribution functions, J. High Energy Phys. 07 (2010) 032.

[96] R. Hamberg, W. L. V. Neerven, and T. Matsuura, A complete calculation of the order $\alpha_{s}^{2}$ correction to the Drell-Yan $K$ factor, Nucl. Phys. B359, 343 (1991); Erratum, Nucl. Phys. B644, 403 (2002).

[97] J. Kubar, M. Le Bellac, J. L. Meunier, and G. Plaut, QCD corrections to the Drell-Yan mechanism and the pion structure function, Nucl. Phys. B175, 251 (1980).

[98] L. H. Song, P. Q. Wang, and Y. J. Zhang, The Drell-Yan nuclear modification due to the nuclear effects of nPDFs and initial-state parton energy loss, Chin. Phys. C 45, 041005 (2021).

[99] C. G. Duan, N. Liu, and Z. Y. Yan, The extraction of nuclear sea quark distribution and energy loss effect in Drell-Yan experiment, Eur. Phys. J. C 50, 585 (2007).

[100] P. Mathews, V. Ravindran, K. Sridhar, and W. L. V. Neerven, Next-to-leading order QCD corrections to the Drell-Yan cross section in models of TeV-scale gravity, Nucl. Phys. B713, 333 (2005).

[101] P. J. Sutton, A. D. Martin, R. G. Roberts, and W. J. Stirling, Parton distributions for the pion extracted from Drell-Yan and prompt photon experiments, Phys. Rev. D 45, 2349 (1992).

[102] S. Catani and M. Grazzini, An NNLO Subtraction Formalism in Hadron Collisions and Its Application to Higgs Boson Production at the LHC, Phys. Rev. Lett. 98, 222002 (2007).

[103] S. Catani, L. Cieri, G. Ferrera, D. de Florian, and M. Grazzini, Vector Boson Production at Hadron Colliders: A Fully Exclusive QCD Calculation at NNLO, Phys. Rev. Lett. 103, 082001 (2009).

[104] M. A. Vasilev et al. (NuSea Collaboration), Parton Energy Loss Limits and Shadowing in Drell-Yan Dimuon Production, Phys. Rev. Lett. 83, 2304 (1999).

[105] D. M. Alde et al., Nuclear Dependence of Dimuon Production at 800-GeV. FNAL-772 Experiment, Phys. Rev. Lett. 64, 2479 (1990).
[106] J. Blumlein, H. Bottcher, and A. Guffanti, Non-singlet QCD analysis of deep inelastic world data at $O\left(\alpha_{s}^{3}\right)$, Nucl. Phys. B774, 182 (2007).

[107] D. Stump, J. Pumplin, R. Brock, D. Casey, J. Huston, J. Kalk, H. L. Lai, and W. K. Tung, Uncertainties of predictions from parton distribution functions. 1. The Lagrange multiplier method, Phys. Rev. D 65, 014012 (2001).

[108] M. Salajegheh, S. M. Moosavi Nejad, H. Khanpour, B. A. Kniehl, and M. Soleymaninia, $B$-hadron fragmentation functions at next-to-next-to-leading order from a global analysis of $e^{+} e^{-}$annihilation data, Phys. Rev. D 99, 114001 (2019).

[109] M. Soleymaninia, M. Goharipour, and H. Khanpour, First QCD analysis of charged hadron fragmentation functions and their uncertainties at next-to-next-to-leading order, Phys. Rev. D 98, 074002 (2018).

[110] F. James and M. Roos, Minuit: A system for function minimization and analysis of the parameter errors and correlations, Comput. Phys. Commun. 10, 343 (1975); F. James, MINUIT function minimization and error analysis: Reference manual version 94.1, Technical Report No. CERN-D-506.

[111] J. Pumplin, D. R. Stump, and W. K. Tung, Multivariate fitting and the error matrix in global analysis of data, Phys. Rev. D 65, 014011 (2001).

[112] J. Pumplin, D. Stump, R. Brock, D. Casey, J. Huston, J. Kalk, H. L. Lai, and W. K. Tung, Uncertainties of predictions from parton distribution functions. 2. The Hessian method, Phys. Rev. D 65, 014013 (2001).

[113] N. P. Hartland, F. Maltoni, E. R. Nocera, J. Rojo, E. Slade, E. Vryonidou, and C. Zhang, A Monte Carlo global analysis of the standard model effective field theory: The top quark sector, J. High Energy Phys. 04 (2019) 100.

[114] A. Buckley, J. Ferrando, S. Lloyd, K. Nordström, B. Page, M. Rüfenacht, M. Schönherr, and G. Watt, LHAPDF6: Parton density access in the LHC precision era, Eur. Phys. J. C 75, 132 (2015).

[115] S. Dulat, T. J. Hou, J. Gao, M. Guzzi, J. Huston, P. Nadolsky, J. Pumplin, C. Schmidt, D. Stump, and C. P. Yuan, New parton distribution functions from a global analysis of quantum chromodynamics, Phys. Rev. D 93, 033006 (2016).

[116] P. Jimenez-Delgado and E. Reya, Dynamical NNLO parton distributions, Phys. Rev. D 79, 074023 (2009).

[117] S.S. Adler et al. (PHENIX Collaboration), Centrality Dependence of $\pi^{0}$ and $\eta$ Production at Large Transverse Momentum in $\sqrt{s_{N N}}=200 \mathrm{GeV} d+\mathrm{Au}$ Collisions, Phys. Rev. Lett. 98, 172302 (2007).

[118] B. I. Abelev et al. (STAR Collaboration), Inclusive $\pi^{0}, \eta$, and direct photon production at high transverse momentum in $p+p$ and $d+\mathrm{Au}$ collisions at $\sqrt{s_{N N}}=200 \mathrm{GeV}$, Phys. Rev. C 81, 064904 (2010).

[119] J. Adams et al. (STAR Collaboration), Identified hadron spectra at large transverse momentum in $\mathrm{p}+\mathrm{p}$ and $\mathrm{d}+\mathrm{Au}$ collisions at $\sqrt{s_{N N}}=200 \mathrm{GeV}$, Phys. Lett. B 637, 161 (2006). 
[120] A. Accardi et al., Electron ion collider: The next QCD frontier: Understanding the glue that binds us all, Eur. Phys. J. A 52, 268 (2016).

[121] P. Agostini et al. (LHeC Collaboration and FCC-he Study Group), The large hadron-electron collider at the HL-LHC, arXiv:2007.14491.
[122] A. Abada et al. (FCC Collaboration), FCC physics opportunities: Future circular collider conceptual design report volume 1, Eur. Phys. J. C 79, 474 (2019).

[123] A. Abada et al. (FCC Collaboration), FCC-hh: The hadron collider: Future circular collider conceptual design report volume 3, Eur. Phys. J. Special Topics 228, 755 (2019). 